\title{
The free compressible viscous vortex
}

\author{
By TIM COLONIUS, SANJIVA K. LELE $\dagger$ \\ AND PARVIZ MOIN \\ Department of Mechanical Engineering, Stanford University, Stanford, CA 94305, USA
}

(Received 22 June 1990 and in revised form 21 December 1990)

\begin{abstract}
The effects of compressibility on free (unsteady) viscous heat-conducting vortices are investigated. Analytical solutions are found in the limit of large, but finite, Reynolds number, and small, but finite, Mach number. The analysis shows that the spreading of the vortex causes a radial flow. This flow is given by the solution of an ordinary differential equation (valid for any Mach number), which gives the dependence of the radial velocity on the tangential velocity, density, and temperature profiles of the vortex; estimates of the radial velocity found by solving this equation are found to be in good agreement with numerical solutions of the full equations. The experiments of Mandella (1987) also report a radial flow in the vortex, but their estimates are much larger than the analytical predictions, and it is found that the flow inferred from the experiments violates the Second Law of Thermodynamics for twodimensional axisymmetric flow. It is speculated that three-dimensionality is the cause of this discrepancy. To obtain detailed analytical solutions, the equations for the viscous evolution are expanded in powers of Mach number, $M$. Solutions valid to $O\left(M^{2}\right)$, are discussed for vortices with finite circulation. Two specific initial conditions - vortices with initially uniform entropy and with initially uniform density - are analysed in detail. It is shown that swirling axisymmetric compressible flows generate negative radial velocities far from the vortex core owing to viscous effects, regardless of the initial distributions of vorticity, density and entropy.
\end{abstract}

\section{Introduction}

Many investigators have studied the two-dimensional axisymmetric vortex at various asymptotic limits of the governing non-dimensional parameters. For inviscid flow, vortex solutions are easily found for compressible or incompressible flows. There is a class of solutions which satisfy

$$
\partial p / \partial r=\rho v^{2} / r
$$

where, $r, p, \rho$, and $v$ are the distance from the centre of the vortex, the pressure, density and tangential velocity respectively. For a compressible vortex, additional information must be supplied to specify the inviscid vortex, because the equations of motion are satisfied for either arbitrary density or temperature profiles. Taylor (1930) considered the inviscid solution for the homentropic case.

For the incompressible viscous vortex in an unbounded domain and with no radial velocity component, no steady solutions are possible. The unsteady equations of motion reduce to

$$
\partial v / \partial t=\nu \partial / \partial r(1 / r \partial / \partial r(r v)), \quad \partial p / \partial r=\rho v^{2} / r .
$$

$\dagger$ Also with Department of Aeronautics and Astronautics, Stanford University.

$\ddagger$ Also with NASA-Ames Research Center. 
Equation $(1.2 a)$ can be transformed into an equation for the vorticity,

$$
\frac{\partial \omega}{\partial t}=\nu\left(\frac{1}{r}\right)\left(\frac{\partial}{\partial r}\left(r \frac{\partial \omega}{\partial r}\right)\right)
$$

which is analogous to the diffusion of heat. The solution to (1.3) can be found for an arbitrary initial distribution of vorticity. De Neufville (1957) has found the solutions of (1.3) as a sum of Laguerre polynomials with argument $r^{2} / 4 v t$ :

$$
\omega(r, t)=\sum_{m=0}^{\infty} C_{m} t^{-(m+1)} \exp \left(\frac{-r^{2}}{4 \nu t}\right) L_{m}\left(\frac{-r^{2}}{4 \nu t}\right), \quad t>0,
$$

where $C_{m}$ are determined from the initial condition by orthogonality of the Laguerre polynomials, $L_{m}$, e.g. Abramowitz \& Stegun (1972). The case $m=0$ corresponds to the solution to (1.2a) found by Oseen (1912),

$$
v=\Gamma / 2 \pi r\left(1-\exp \left(-r^{2} / 4 v t\right)\right)
$$

which corresponds to the decay of a Gaussian vorticity distribution, or the viscous spreading of a line vortex. $\Gamma$ is the circulation at infinity, which is an invariant of the flow. The $m=1$ case corresponds to a solution found by Taylor (1918),

$$
v=\frac{\mathscr{M} r}{16 \pi v^{2} t^{2}} \exp \left(\frac{-r^{2}}{4 v t}\right)
$$

The circulation of the Taylor swirl is zero, and the invariant of the flow is

$$
\mathscr{M}=\int_{0}^{\infty} 2 \pi r v r \mathrm{~d} r
$$

which is the total angular momentum of the flow (the density has been taken to be uniform).

Some solutions for the case of steady, viscous, compressible vortices have been found. For example, the solution of Mack (1960) describes a compressible vortex which is driven by a solid inner cylinder. Steady state is obtained by equating the work done by the shear stress and the heat transferred by conduction. Mack found that this solution is not homentropic. Mack's solution is extended to the case of nonzero radial velocity (porous inner cylinder) corresponding to a steady, spiralling flow by Bellamy-Knights (1980). Rott (1959) discusses the temperature distributions for the (steady) Burgers vortex at low Mach number. Sibulkin (1961) discusses the energy transfer associated with the decay of the Taylor swirl for both liquids and gases in the low-Mach-number limit. For unsteady, viscous, compressible vortices, theoretical predictions for the density profile in the compressible vortex core produced by shock tube flow over a vertical wedge are given by Merzkirch (1964).

Analytical solutions for the case of a free (unsteady), viscous, compressible vortex apparently have not been reported. The purpose of this paper is to investigate the effects of compressibility of the free, viscous, heat-conducting vortex. The experiments of Mandella, Moon \& Bershader (1986), Mandella (1987), and Bershader (1988) (collectively referred to as $M \& B$ hereinafter) examine the structure of compressible vortices. They report that the density and pressure within the core of the vortex is considerably lower than ambient values, and infer that the spreading of the vortex causes a significant inward radial velocity. The present work is motivated by these experiments and numerical solutions of the full compressible Navier-Stokes equations performed by the present authors. The numerical solutions 
also generate a radial inflow, but its magnitude is $\mathbf{1 0}$ to 100 fold smaller than that inferred by the experimental study.

The presence of a radial component of velocity causes angular momentum to be convected into the vortex, counteracting the effect of viscous diffusion of angular momentum. The magnitude of the radial velocity in the vortex is, therefore, important in determining the balance between the opposing effects of viscous diffusion and radial convection of angular momentum. The magnitude of the radial velocity in the vortex is also related to the rate of change of density in the vortex. A vortex with an initially lower density in the core must gain mass as it decays to ambient conditions, which results in a negative radial velocity. Since the pressure in the core of the vortex is lower than ambient, a homentropic vortex would also have lower density in the core, and thus a negative radial velocity. If the vortex remains homentropic, then the pressure and density must both increase with time. M \& B found that the vortex is not homentropic, but that the density is indeed much lower in the core than in the surroundings. From measured density and pressure distributions in the core of the vortex, they infer the radial velocity profiles and find that the effect of convection of angular momentum is larger than the effect of outward diffusion of angular momentum.

This paper presents analytical solutions for free compressible viscous vortices, and compares them to numerical solutions of the full equations as well as the experiments of $M \& B$. The discrepancy between the experiments and the full solutions is directly addressed and is attributed to three-dimensional effects in the experiments. It is speculated that similar three-dimensionality may be present in other experiments involving 'two-dimensional' compressible vortices.

In $\S 2$ the full equations are presented. In $\S 3$, these equations are expanded for high Reynolds number, and on two timescales, viz. an acoustic time and a viscous time. An ordinary differential equation for the radial velocity is derived, and solutions of this equation are compared to numerical computations and the aforementioned experiments. In $\$ 4$ the equations for evolution on the viscous timescale are expanded in powers of $M^{2}$ where $M$ is the Mach number. Analytical solutions for the $O(1)$, and $O\left(M^{2}\right)$ equations are found for particular initial conditions. A summary of the conclusions is given in $\$ 5$.

\section{Governing equations}

For two-dimensional axisymmetric flow, the equations of continuity, conservation of momentum and energy, and an equation of state (perfect gas) are

$$
\begin{aligned}
& \frac{\partial \hat{\rho}}{\partial \hat{t}}+\frac{1}{\hat{r}} \frac{\partial(\hat{\rho} \hat{u} \hat{r})}{\partial \hat{r}}=0, \\
& \hat{\rho}\left(\frac{\partial \hat{u}}{\partial \hat{t}}+\hat{u} \frac{\partial \hat{u}}{\partial \hat{r}}-\frac{\hat{v}^{2}}{\hat{r}}\right)=-\frac{\partial \hat{p}}{\partial \hat{r}}+\frac{4}{3 R e} \frac{\partial}{\partial \hat{r}}\left(\frac{1}{\hat{r}} \frac{\partial}{\partial \hat{r}}(\hat{r} \hat{u})\right), \\
& \hat{\rho}\left(\frac{\partial \hat{v}}{\partial \hat{t}}+\hat{u} \frac{\partial \hat{v}}{\partial \hat{r}}+\frac{\hat{v} \hat{u}}{\hat{r}}\right)=\frac{1}{R e} \frac{\partial}{\partial \hat{r}}\left(\frac{1}{\hat{r}} \frac{\partial}{\partial \hat{r}}(\hat{r} \hat{v})\right), \\
& \frac{\partial \hat{p}}{\partial \hat{t}}+\hat{u} \frac{\partial \hat{p}}{\partial \hat{r}}+\gamma \hat{p}\left(\frac{\partial \hat{u}}{\partial \hat{r}}+\frac{\hat{u}}{\hat{r}}\right)=\frac{(\gamma-1)}{\operatorname{RePr}} \frac{1}{\hat{r}} \frac{\partial}{\partial \hat{r}}\left(\hat{r} \frac{\partial \hat{T}}{\partial \hat{r}}\right)+\frac{2(\gamma-1)}{\operatorname{Re}} \hat{\boldsymbol{\Phi}}, \\
& \hat{p}=\frac{(\gamma-1)}{\gamma} \hat{\rho} \hat{T},
\end{aligned}
$$


where $\hat{u}$ and $\hat{v}$ are the radial and tangential velocities respectively, and

$$
\hat{\Phi}=\left(\frac{\partial \hat{u}}{\partial \hat{r}}\right)^{2}+\left(\frac{\hat{u}}{\hat{r}}\right)^{2}-\frac{1}{3}\left(\frac{\partial \hat{u}}{\partial \hat{r}}+\frac{\hat{u}}{\hat{r}}\right)^{2}+\frac{1}{2}\left(\frac{\partial \hat{v}}{\partial \hat{r}}-\frac{\hat{v}}{\hat{r}}\right)^{2}
$$

and where the following non-dimensionalization has been used:

$$
\left.\begin{array}{c}
\hat{r}=\frac{r}{L}, \quad \hat{t}=\frac{a_{\infty} t}{L}, \quad \hat{v}=\frac{v}{a_{\infty}}, \quad \hat{u}=\frac{u}{a_{\infty}}, \\
\hat{\rho}=\frac{\rho}{\rho_{\infty}}, \quad \hat{p}=\frac{p}{\rho_{\infty} a_{\infty}^{2}}, \quad \hat{T}=\frac{c_{p} T}{a_{\infty}^{2}} .
\end{array}\right\}
$$

The Reynolds number and Prandtl number are defined as

$$
R e=\frac{\rho_{\infty} a_{\infty} L}{\mu}, \quad \operatorname{Pr}=\frac{\mu c_{p}}{k} .
$$

In the above, $a_{\infty}$ is the ambient sound speed, $L$ is some lengthscale of the vortex, such as the size of the vortex core. For simplicity, $\mu$ and $k$ are taken to be the constant molecular viscosity and thermal conductivity of the fluid respectively, $c_{p}$ is the specific heat at constant pressure which is assumed constant, and $R$ is the gas constant.

In addition to (2.1), boundary and initial conditions are required for solutions. The flow domain extends to infinity, and therefore solutions are required to be bounded as $\hat{r} \rightarrow \infty$. Furthermore, axisymmetry requires that both components of velocity are zero at $\hat{r}=\mathbf{0}$, and that $\partial \hat{p} / \partial \hat{r}, \partial \hat{T} / \partial \hat{r}$ and $\partial \hat{\rho} / \partial \hat{r}$ all vanish as $\hat{r} \rightarrow 0$. Initial conditions are discussed in $\$ 4$.

General solutions to the nonlinear equations (2.1) are difficult to find, and one is restricted to solving them numerically, or finding asymptotic solutions for small or large values of the parameters.

\section{Multiple timescale expansion}

For large Reynolds number, unsteady solutions to (2.1) can vary on three distinct timescales - acoustic, convective and viscous. For two-dimensional axisymmetric flow, quantities are convected by the radial velocity alone, and if the radial velocity is small compared to the tangential velocity, then convection effects are of secondary importance. Furthermore, for the present problem, the convecting velocity is set up by viscous effects thus making the convective and viscous scales the same. To examine the evolution of the vortex on the viscous timescale, we expand (2.1) on two timescales - the fast acoustic time, and the slow viscous time. Let

$$
\hat{f}=\hat{f}(\hat{t}, \tau, \hat{r}),
$$

where $\hat{f}$ is any of $\hat{u}, \hat{v}, \hat{\rho}, \hat{p}$, or $\hat{T}$, and where,

$$
\tau=\hat{t} / R e .
$$

We require that the radial velocity, $\hat{u}$, is small, and since $\hat{u}$ must vanish for an inviscid vortex as well as for an incompressible vortex, we assume that

$$
\hat{u} \sim 1 / R e .
$$


Expanding (2.1) using (3.1)-(3.3) gives, to $O(1)$,

$$
\frac{\partial \hat{\rho}}{\partial \hat{t}}=\mathbf{0}, \quad \frac{\hat{\rho} \hat{v}^{2}}{\hat{r}}=\frac{\partial \hat{p}}{\partial \hat{r}}, \quad \frac{\partial \hat{v}}{\partial \hat{t}}=\mathbf{0}, \quad \frac{\partial \hat{p}}{\partial \hat{t}}=0, \quad \hat{p}=\frac{(\gamma-1)}{\gamma} \hat{\rho} \hat{T} .
$$

Equations (3.4) govern the evolution of an inviscid compressible vortex. The choice of scaling for $\hat{u}$ gives the desired result of forcing the solutions not to vary on the acoustic timescale. Furthermore, the particular dependence of $R e$ chosen in (3.3) gives good agreement with numerical solutions to $(2.1)$ over a large range of Reynolds number. The numerical solutions will be described in detail in $\$ 3.1$.

Collecting terms of the order $1 / R e$ in the expansion of (2.1) gives

$$
\begin{gathered}
\frac{\partial \hat{\rho}}{\partial \tau}+\frac{1}{\hat{r}} \frac{\partial\left(\hat{\rho} \hat{u}^{*} \hat{r}\right)}{\partial \hat{r}}=0 \\
\hat{\rho}\left(\frac{\partial \hat{v}}{\partial \tau}+\hat{u}^{*} \frac{\partial \hat{v}}{\partial \hat{r}}+\frac{\hat{v} \hat{u}^{*}}{\hat{r}}\right)=\frac{\partial}{\partial \hat{r}}\left(\frac{1}{\hat{r}} \frac{\partial}{\partial \hat{r}}(\hat{r} \hat{v})\right) \\
\frac{\partial \hat{p}}{\partial \tau}+\hat{u}^{*} \frac{\partial \hat{p}}{\partial \hat{r}}+\gamma \hat{p}\left(\frac{\partial \hat{u}^{*}}{\partial \hat{r}}+\frac{\hat{u}^{*}}{\hat{r}}\right)=\frac{(\gamma-1)}{P r} \frac{1}{\hat{r}} \frac{\partial}{\partial \hat{r}}\left(\hat{r} \frac{\partial \hat{T}}{\partial \hat{r}}\right)+(\gamma-1)\left(\frac{\partial \hat{v}}{\partial \hat{r}}-\frac{\hat{v}}{\hat{r}}\right)^{2}
\end{gathered}
$$

and

$$
\frac{\partial \hat{u}^{*}}{\partial \hat{t}}=0
$$

where $\hat{u}^{*}=\operatorname{Re} \hat{u}$.

Equations (3.5) together with (3.4b) and (3.4e), describe the evolution of $\hat{u}^{*}, \hat{v}, \hat{\rho}$, and $\hat{p}$ on the slow time, $\tau$.

If $(3.4 b)$ is differentiated with respect to $\tau$, and $(3.5 a-c)$ are substituted into the resulting expression, an equation for the evolution of $\hat{u}^{*}$ in terms of the other variables is obtained:

$$
\begin{aligned}
\frac{\partial^{2} \hat{u}^{*}}{\partial \hat{r}^{2}}+\left(\frac{1}{\hat{r}}+\frac{\gamma \hat{v}^{2}}{\hat{a}^{2} \hat{r}}\right) \frac{\partial \hat{u}^{*}}{\partial \hat{r}}+\left(\frac{(\gamma-4) \hat{v}^{2}}{\hat{a}^{2} \hat{r}^{2}}-\frac{1}{\hat{r}^{2}}\right) \hat{u}^{*}=\frac{-2 \hat{v}}{\hat{a}^{2} \hat{\rho} \hat{r}} \frac{\partial}{\partial \hat{r}}\left(\frac{1}{\hat{r}} \frac{\partial}{\partial \hat{r}}(\hat{r} \hat{v})\right) \\
+\frac{(\gamma-1)}{\operatorname{Pr} \hat{\rho} \hat{a}^{2}} \frac{\partial}{\partial \hat{r}}\left(\frac{1}{\hat{r}} \frac{\partial}{\partial \hat{r}}\left(\hat{r} \frac{\partial \hat{T}}{\partial \hat{r}}\right)\right)+\frac{(\gamma-1)}{\hat{\rho} \hat{a}^{2}} \frac{\partial}{\partial \hat{r}}\left(\frac{\partial \hat{v}}{\partial \hat{r}}-\frac{\hat{v}}{\hat{r}}\right)^{2},
\end{aligned}
$$

where $\hat{a}$ is the sound speed relative to $a_{\infty}$. This is an ordinary differential equation for $\hat{u}^{*}$, in terms of the profiles of $\hat{v}, \hat{\rho}$, and $\hat{T}$. This equation is expected to hold for any Mach number provided that the Reynolds number is sufficiently high.

\subsection{Comparison with experiments and computations}

Equations (3.4e), (3.5 $a-c)$, and (3.7) are still difficult to solve analytically. Equation (3.7) expresses the dependence of the radial velocity induced by the spreading of the vortex on the temperature, density and tangential velocity profiles. Note that there is no explicit time dependence in (3.7), hence it may be solved as an ordinary differential equation. Physically, (3.7) gives the necessary radial velocity such that, to first order, the centripetal acceleration in the vortex is provided by the radial pressure gradient, i.e. $(3.4 b)$ is satisfied. The three terms on the right-hand side of (3.7) represent viscous diffusion of momentum, heat conduction, and viscous dissipation, respectively. The radial velocity of the vortex is an effect of compressibility (since a strictly incompressible vortex must have zero radial velocity); however, it is forced by viscous and heat conduction effects as given by 

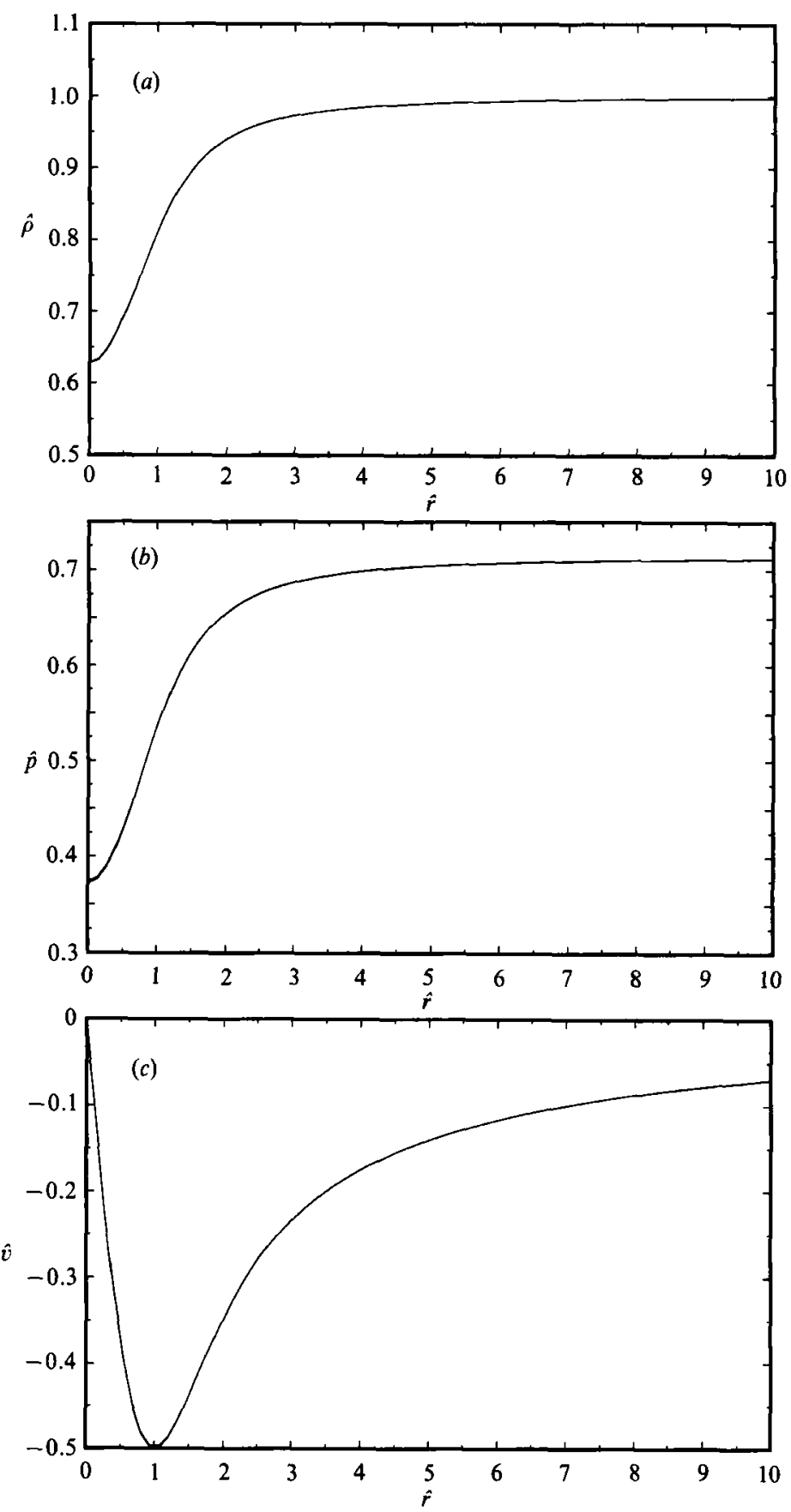

FIoUre $1(a-c)$. For caption see facing page.

(3.7). Note that because of the multiple timescale expansions, (3.4), (3.5a-c) and (3.7) are valid only after initial acoustic disturbances have propagated away from the vortex, since general initial conditions to (2.1) will produce an acoustic wave which propagates away from $\hat{r}=0$ at the sonic velocity. At any radial location, the equations are valid if $\hat{r} \ll \hat{a t}$. 


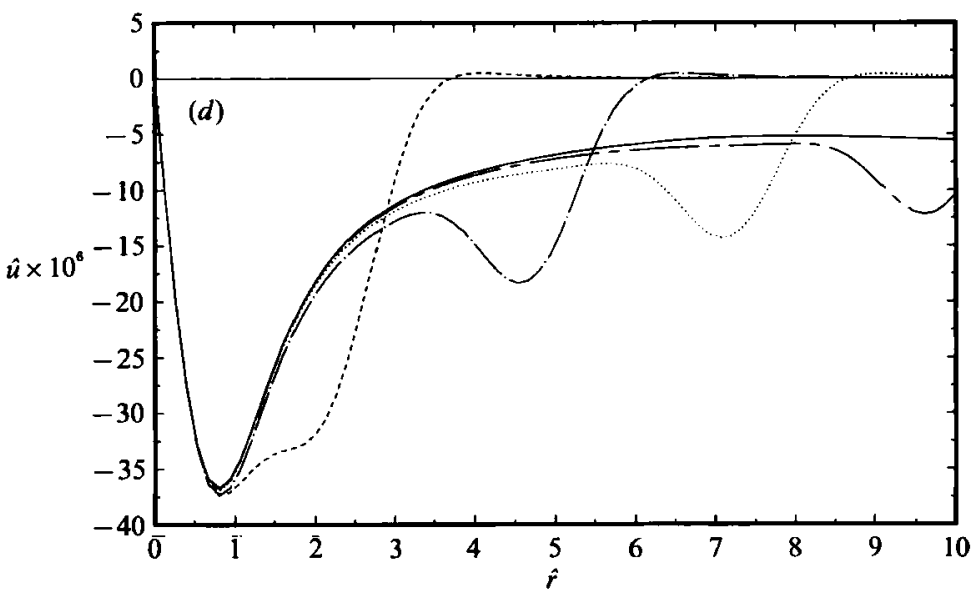

Figure 1. Evolution of $(a)$ density, $(b)$ pressure, $(c)$ tangential velocity, and $(d)$ radial velocity from computations at six times during acoustic transient. Initial condition $(\hat{t}=0)$ is inviscid and homentropic. $\overline{R e}=5000, M=0.5:-, \hat{t}=0 ;----, \hat{t}=2.5 ;---, \hat{t}=\mathbf{5 . 0} ; \cdots \cdots \cdots, \hat{t}=7.5$; ,$-- \hat{t}=10.0 ;-, \hat{t}=12.5$.

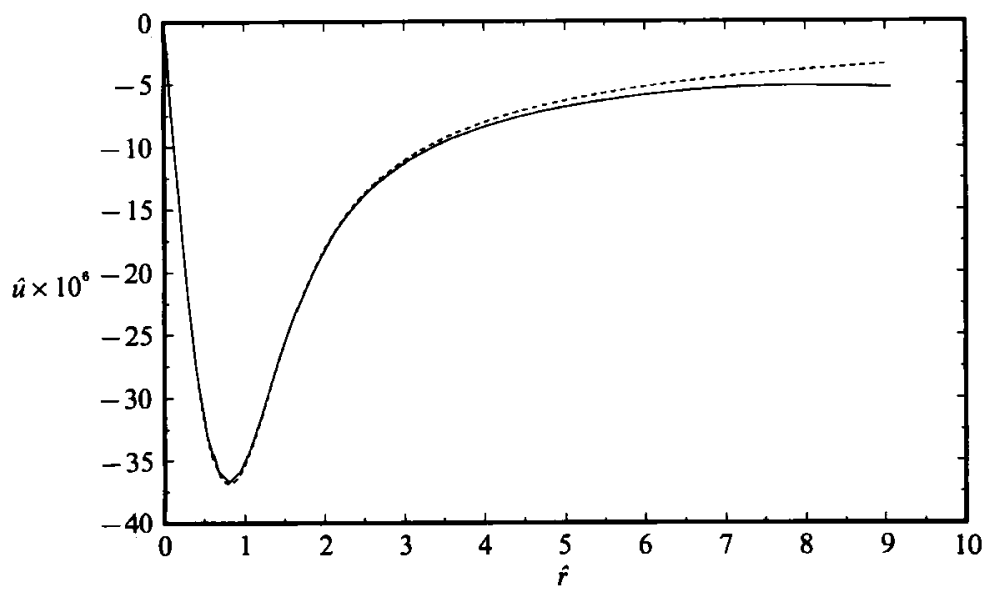

Ftgure 2. Comparison of radial velocity from the computed full solution (same condition as in figure $1, \hat{t}=12.5$ ) and radial velocity computed from equation $(3.7):-$, simulation after acoustic transient; ----, numerical solution of (3.7).

The validity of (3.7) is checked by comparing its numerical solution with numerical solutions of the full equations (2.1). For solving the full compressible equations a grid of 151 mesh points extending to \pm 10 core radii in each spatial dimension is used. A sixth-order modified Padé scheme (Lele 1990) is used to compute spatial derivatives, and the equations are advanced with a fourth-order Runge-Kutta scheme. Non-reflecting boundary conditions (Thompson 1987 and Colonius, Lele \& Moin 1991) are used at the computational boundaries. At the boundary, the conservation equations are solved in characteristic form (e.g. Thompson 1987) where terms representing the amplitude of different waves crossing the boundary are isolated. These characteristic amplitudes are then specified either by computation from interior grid points via one-sided differences if the wave is propagating out of the domain, or are specified independently of the interior numerical solution for waves propagating into the domain. In the absence of a detailed knowledge of the 

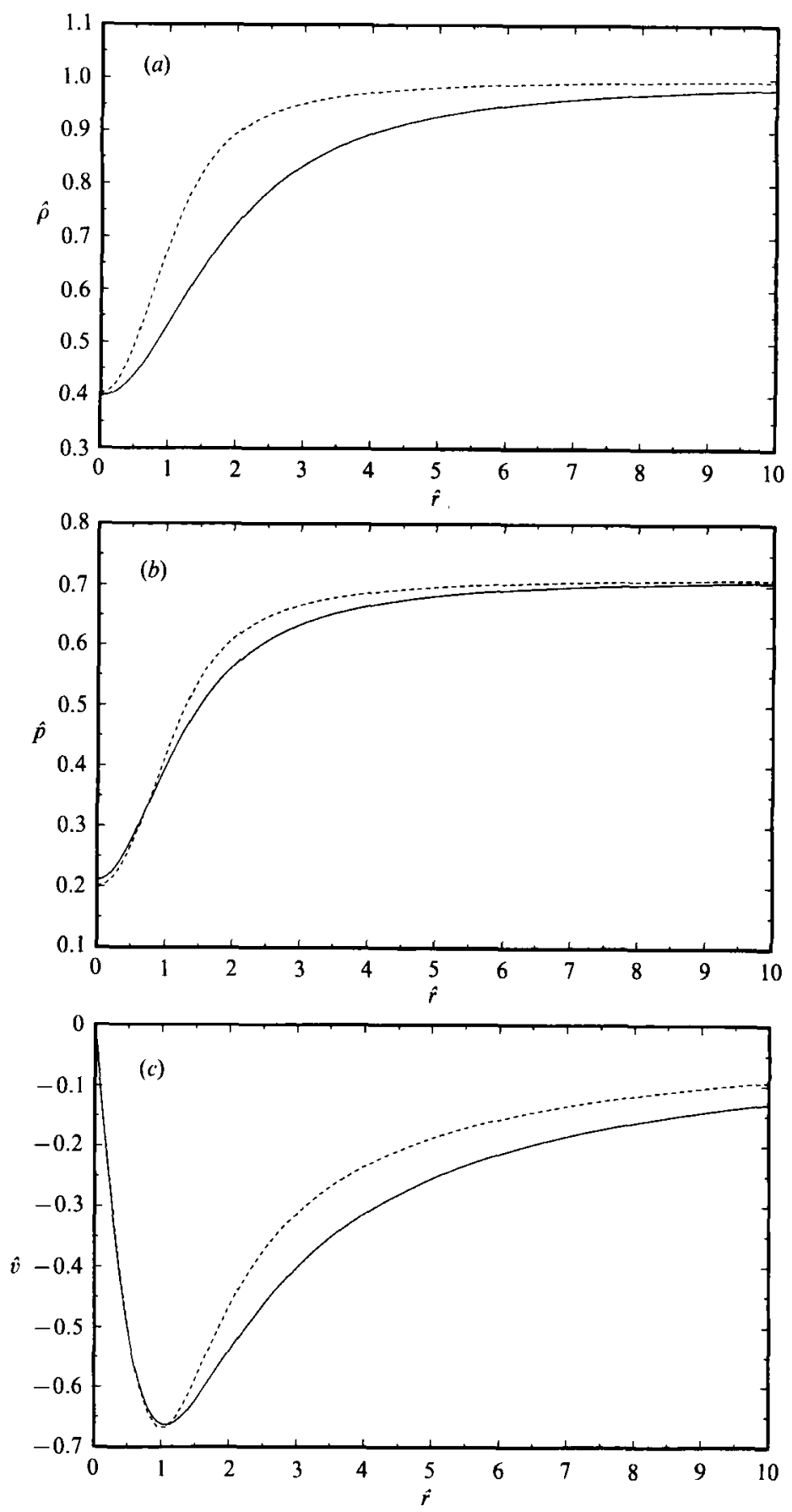

Figure $3(a-c)$. For caption see facing page.

flow field outside the computational domain, one specifies the amplitudes of the incoming characteristic waves to be zero for all times (Thompson 1987). In the case of the vortex, the mean flow gradients are not zero at the boundary, and such boundary specifications are not appropriate. Since the vortex flow is irrotational outside the core, the mean flow at the boundary is essentially inviscid and steady 


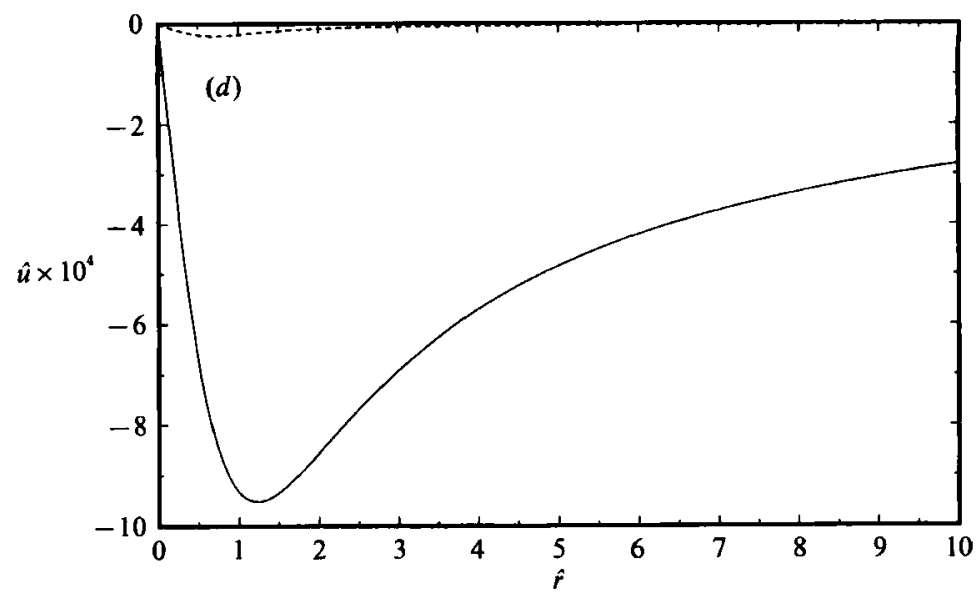

Figure 3. Comparison of $(a)$ density, $(b)$ pressure, $(c)$ tangential velocity, and $(d)$ radial velocity from computations and experiments of Mandella (1987). $\overline{R e}=20800, M=0.67$ : —, data of Mandella (1987);----, numerical solution.

until the viscous vortex core has spread to near the boundary. The amplitudes of the incoming characteristics are then approximated with the gradients of the homentropic, irrotational initial condition at the boundary. We note that the resulting boundary conditions are not perfectly non-reflecting to acoustic disturbances, but reasonable accuracy near the computational boundaries is verified by performing computations on different-sized computational domains (Colonius et al. 1991), and errors due to the boundary conditions are localized near the computational boundary.

The computations are initialized with an inviscid solution to (1.1). The initial vorticity profile is assumed to be Gaussian, and homentropic. The radial velocity is initially set to zero. Since the pressure is initially lower than ambient in the core of the vortex, the density is also lower than ambient; for $M=v_{\mathrm{m}} / a_{\infty}>1.211$ (when $\gamma$ $=1.4$ ) (where $v_{\mathrm{m}}$ is the maximum tangential velocity and $a_{\infty}$ the ambient sonic velocity) the vortex core is evacuated in the initial condition. (In terms of the maximum local Mach number, $M^{*}=v_{\mathrm{m}} / a_{\mathrm{m}}>2.276$ for evacuation of the core.)

Since the initial condition is not a solution to the viscous equations $(2.1)$, there is a transient period in which an acoustic wave is generated at the vortex centre and travels radially outward at the speed of sound. Figure 1 shows the evolution of the density, pressure, tangential velocity and radial velocities during the acoustic transient. The Reynolds number is sufficiently high $\left(\overline{R e}=\rho_{\infty} v_{\mathrm{m}} L / \mu=5000\right.$, where $L$ is the radial position where the tangential velocity attains a maximum, $v_{\mathrm{m}}$ ) that the change in the pressure, density, and tangential velocity over the acoustic timescale is negligible. However, the radial velocity adjusts from zero to the profile shown in the figure. All variables in the figures are made non-dimensional as in (2.3). In figure 2 the quasi-steady radial velocity obtained from the full solution is compared to that predicted by (3.7). For this comparison, (3.7) is solved numerically for the given pressure, density and tangential velocity profiles of figure 1 . The agreement is good, except near $\hat{r}=10$, where the numerical solution diverges from the solution of (3.7). This discrepancy is due to small errors in the numerical solutions at the artificial numerical boundary located at $\hat{r}=10$, due to the approximations mentioned above. This procedure is repeated for several Reynolds numbers, and the agreement is quantitatively accurate to within $10 \%$ for $\overline{R e}>1000$. Only qualitative 


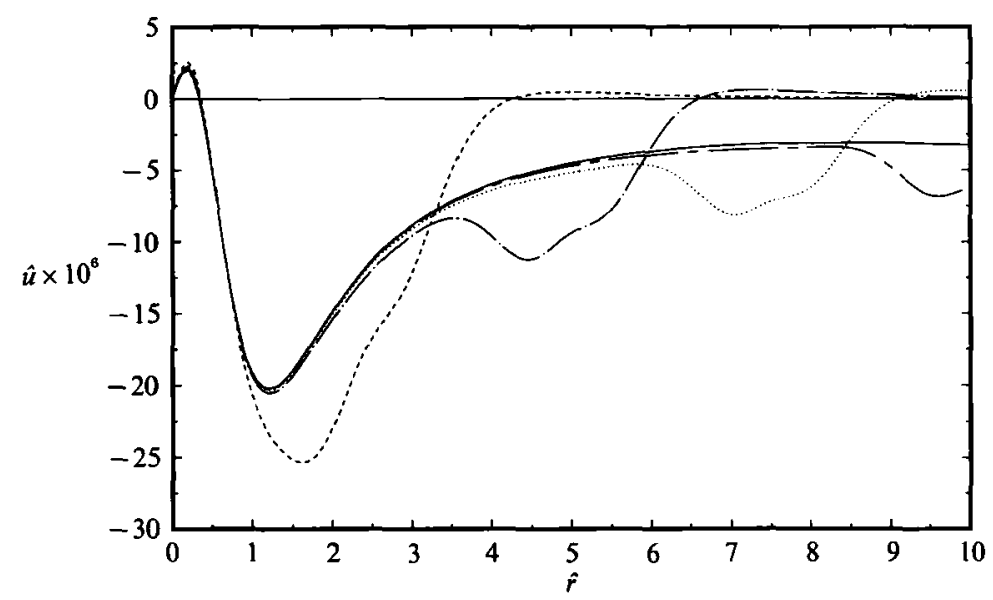

Figure 4. Evolution of radial velocity from computation. Initial condition $(\hat{t}=0)$ for density pressure and tangential velocity from Mandella (1987), radial velocity is initially zero. $\overline{R e}=20800$, $M=0.67:-, \hat{t}=0 ;---, \hat{t}=2.5 ;-\cdots, \hat{t}=5.0 ; \cdots \cdots, \hat{t}=7.5 ;-\cdots, \hat{t}=10.0 ;-, \hat{t}=$ 12.5 .

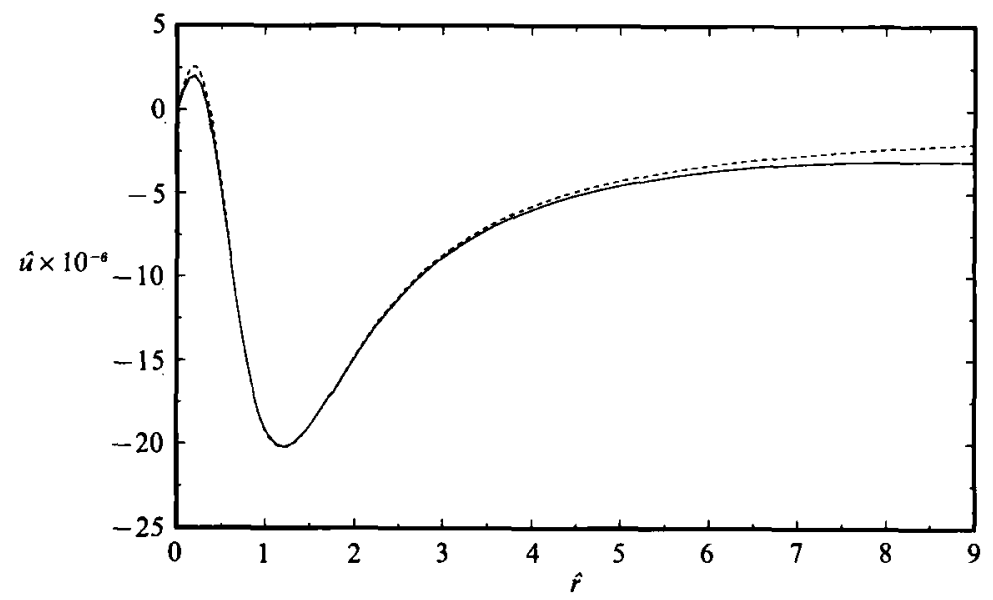

Figure 5. Comparison of radial velocity of computed vortex of figure 4 at $\hat{t}=12.5$ and radial velocity computed from (3.7):- - , simulation after acoustic transient; -----, numerical solution of (3.7).

agreement is observed for Reynolds numbers as low as 100. Similarly, (3.7) is solved over a range of Mach numbers $\left(M=v_{\mathrm{m}} / a_{\infty}\right)$ from 0.0625 to 0.67 , and the agreement is good over the entire range.

Experiments performed by M \& B, however, show a much larger radial velocity than predicted by the numerical solutions, and estimated by (3.7). In the experiments of $M \& B$ a shock wave is passed over an airfoil at large angle of attack. The resulting starting vortex is convected downstream of the airfoil. Measurements of the density and pressure distributions inside the vortex were made; the density was measured by the method of double-pulsed holographic interferometry, and the pressure was measured by a pressure transducer mounted flush on one of the sidewalls of the test section. The measured density and pressure distributions are used to infer values of the radial and tangential velocity profiles through the use of the continuity equation (equation $(2.1 a)$ ), and (3.4b), respectively. Figure 3 shows the distribution of the 


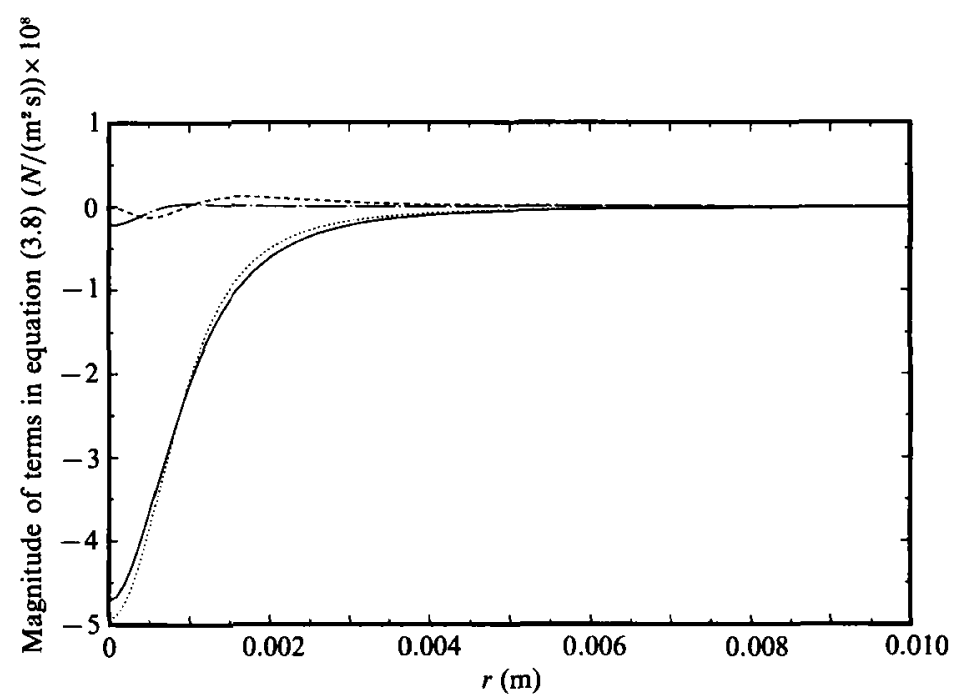

Figure 6. Balance of terms in the Second Law of Thermodynamics, equation (3.8), computed from data of Mandella (1987): -,$\rho T \partial s / \partial t ;----, \rho T u \partial s / \partial r ;-.-,(1 / r)(\partial / \partial r) r k \partial T / \partial r ; \cdots \cdots$, sum of terms.

density, pressure, and velocities at one measurement time $(t=500 \mu \mathrm{s}, \hat{t}=191)$ from the experiment. The Reynolds number $\widetilde{R e}$ and Mach number are 20800 and 0.67 respectively at this measurement time. Superimposed on these distributions are computational results for an initially homentropic vortex at the same Reynolds and Mach numbers. The computed vortex which is plotted on the figure has undergone the acoustic transient described above. The distributions of the pressure, density, and tangential velocity are qualitatively similar to the experimental vortex. However, the radial velocity inferred from the experimental data is two orders of magnitude larger than the computed radial velocity. (The radial velocity compared to the tangential velocity inferred from the experiment and from the full solution are $10^{-5}$ and $10^{-7}$, respectively.) The difference between the distribution of density, pressure, and tangential velocities between the experiments and computations is at most $50 \%$, and cannot account for a 100 fold change in radial velocity.

This is borne out by computations where the initial condition for the pressure, density, and tangential velocity are taken from the experiments, whereas the initial radial velocity was set to zero. Figure 4 shows the distribution of radial velocity during the acoustic transient. The computed values of $\hat{v}, \hat{p}$, and $\hat{\rho}$ do not change significantly. During a similar time period $M \& B$ report a significant change in the measured values of $\hat{\rho}$. Figure 5 shows the resulting radial velocity distribution from the computations compared with the profile predicted by (3.7) solved using for the experimental distributions of density, pressure, and tangential velocity. Again the agreement is good. However, the radial velocity predicted by the computation and (3.7) is still two orders of magnitude smaller than that inferred by the solution of the continuity equation from the measured distributions of density.

Is it possible that both radial velocity distributions are correct, since the solutions depend on initial conditions? To address this issue, a computation is conducted where the initial conditions for all four variables (density, pressure, tangential and radial velocities) are taken from the experiments. During the time interval computed (corresponding approximately to the length of time in the experiment) the pressure, 
density, and tangential velocity decay negligibly, while the radial velocity undergoes a large transient. The values of the radial velocity after the transient wave leaves the computational domain $(\hat{t}=12.5)$ are two orders of magnitude smaller than the initial distribution $(\hat{t}=0)$.

To further investigate this inconsistency, the Second Law of Thermodynamics is employed. For two-dimensional axisymmetric flow, in dimensional form, the Second Law is

$$
\rho T \partial s / \partial t+\rho T u \partial s / \partial r-1 / r \partial / \partial r(r k \partial T / \partial r) \geqslant 0
$$

where $s$ is the entropy. Equation (3.8) is exact; the inequality results from the positive definite viscous dissipation associated with the motion. Ideal gas relations yield

$$
\frac{\partial s}{\partial r}=\frac{c_{v}}{p} \frac{\partial p}{\partial r}-\frac{c_{p}}{\rho} \frac{\partial \rho}{\partial r}, \quad \frac{\partial s}{\partial t}=\frac{c_{v}}{p} \frac{\partial p}{\partial t}-\frac{c_{p}}{\rho} \frac{\partial \rho}{\partial t} .
$$

And with the use of $(3.4 b)$, which $M \& B$ use to determine the tangential velocity from the measured pressure profile,

$$
\frac{\partial p}{\partial t}=-\int_{r}^{\infty}\left[\frac{v^{2}}{r} \frac{\partial \rho}{\partial t}+\frac{2 \rho v}{r} \frac{\partial v}{\partial t}\right] \mathrm{d} r
$$

All of the quantities on the right-hand sides of (3.9) and (3.10) are computed from the experimental data reported by $M \& B$. The density, radial velocity and temperature distributions in (3.8) are also computed from the experimental data. The conductivity, $k$, is assumed to vary linearly with temperature over the range of temperatures in the experiments (Incropera \& DeWitt 1985). These terms are then substituted into the Second Law (3.8). Each of the three terms on the left-hand side of (3.8) is plotted in figure 6, as well as the sum of the three terms. Since the sum is less than zero for all $\hat{r}$, the distributions of density, pressure, and radial and tangential velocity measured and inferred in the experiments are not valid for twodimensional axisymmetric flow since they violate the Second Law.

The experimental vortex could not have been two-dimensional and axisymmetric. The measured rate of change of density cannot be only due to the radial velocity. In the framework of (3.7), viscous effects alone cannot generate a radial velocity large enough to balance the measured rate of change of density. The presence of a third velocity component (along the vortex axis) is a possible explanation for the discrepancy. The experimental vortex could have been influenced by the boundary layers on the sidewalls of the wind tunnel. Rott \& Lewellen (1966) show that the flow induced into the boundary layer by the vortex may be sufficiently large to have a dominant role in the conservation of angular momentum in the vortex. We speculate that similar deviations from two-dimensionality may be present in other 'twodimensional' experiments involving compressible vortices.

Equation (3.7) is useful in estimating the magnitude of the radial velocity in the vortex given the distributions of tangential velocity and temperature. However, general solutions to $(3.4 e),(3.5 a-c)$, and (3.7) are still difficult to find. In the next section we expand these equations in powers of $M^{2}$ and find exact solutions for the $O(1)$ base flow and the $O\left(M^{2}\right)$ perturbations.

\section{Low-Mach-number expansion}

In $\S 3$ the full equations for two-dimensional axisymmetric flow of an ideal gas (equations (2.1)) were expanded on two timescales, an acoustic time and a viscous 
time. The evolution of the density, tangential velocity, radial velocity, and pressure on the viscous timescale are given by $(3.4 e),(3.5 a-c)$, and (3.7). These equations are now expanded in powers of Mach number squared. First the equations are rewritten using the following (incompressible) non-dimensionalization:

$$
\left.\begin{array}{c}
\tilde{r}=\frac{r}{L}, \quad \tilde{t}=\frac{v_{\mathrm{m}} t}{L}=\overline{R e} \tau, \quad \tilde{v}=\frac{v}{v_{\mathrm{m}}}, \quad \tilde{u}=\frac{u}{v_{\mathrm{m}}} \\
\tilde{\rho}=\frac{\rho}{\rho_{\infty}}, \quad \tilde{p}=\frac{p-p_{\infty}}{\rho_{\infty} v_{\mathrm{m}}^{2}}, \quad \tilde{T}=\frac{T}{T_{\infty}}, \quad \tilde{a}=\frac{a}{a_{\infty}}
\end{array}\right\}
$$

and

$$
\overline{R e}=\frac{\rho_{\infty} v_{\mathrm{m}} L}{\mu}, \quad \operatorname{Pr}=\frac{\mu c_{p}}{k}, \quad M=\frac{v_{\mathrm{m}}}{a_{\infty}},
$$

where $L$ is again some lengthscale of the vortex such as the core size, and $v_{\mathrm{m}}$ is the reference tangential velocity, $v$. We obtain

$$
\begin{gathered}
\frac{\partial \tilde{\rho}}{\partial \tilde{t}}+\frac{1}{\tilde{r}} \frac{\partial(\tilde{\rho} \tilde{u} \tilde{r})}{\partial \tilde{r}}=0 \\
\tilde{\rho}\left(\frac{\partial \tilde{v}}{\partial \tilde{t}}+\tilde{u} \frac{\partial \tilde{v}}{\partial \tilde{r}}+\frac{\tilde{v} \tilde{u}}{\tilde{r}}\right)=\frac{1}{\overline{R e}} \frac{\partial}{\partial \tilde{r}}\left(\frac{1}{\tilde{r}} \frac{\partial}{\partial \tilde{r}}(\tilde{r} \tilde{v})\right) \\
\frac{\partial^{2} \tilde{u}}{\partial \tilde{r}^{2}}+\left(\frac{1}{\tilde{r}}+\frac{\gamma M^{2} \tilde{v}^{2}}{\tilde{a}^{2} \tilde{r}}\right) \frac{\partial \tilde{u}}{\partial \tilde{r}}+\left(\frac{(\gamma-4) M^{2} \tilde{v}^{2}}{\tilde{a}^{2} \tilde{r}^{2}}-\frac{1}{\tilde{r}^{2}}\right) \tilde{u}=\frac{-2 \tilde{v} M^{2}}{\operatorname{Re} \tilde{a}^{2} \tilde{\rho} \tilde{r}} \frac{\partial}{\partial \tilde{r}}\left(\frac{1}{\tilde{r}} \frac{\partial}{\partial \tilde{r}}(\tilde{r} \tilde{v})\right) \\
+\frac{1}{\overline{R e} \operatorname{Pr} \tilde{\rho} \tilde{a}^{2}} \frac{\partial}{\partial \tilde{r}}\left(\frac{1}{\tilde{r}} \frac{\partial}{\partial \tilde{r}}\left(\tilde{r} \frac{\partial \tilde{T}}{\partial \tilde{r}}\right)\right)+\frac{(\gamma-1) M^{2}}{\tilde{R e} \tilde{\rho} \tilde{a}^{2}} \frac{\partial}{\partial \tilde{r}}\left(\frac{\partial \tilde{v}}{\partial \tilde{r}}-\frac{\tilde{v}}{\tilde{r}}\right)^{2} \\
\frac{\partial \tilde{T}}{\partial \tilde{t}}+\tilde{u} \frac{\partial \tilde{T}}{\partial \tilde{r}}+(\gamma-1) \tilde{T}\left(\frac{\partial \tilde{u}}{\partial \tilde{r}}+\frac{\tilde{u}}{\tilde{r}}\right)=\frac{\gamma}{\overline{R e} \operatorname{Pr} \tilde{\rho}} \frac{1}{\tilde{r}} \frac{\partial}{\partial \tilde{r}}\left(\tilde{r} \frac{\partial \tilde{T}}{\partial \tilde{r}}\right)+\frac{M^{2} \gamma(\gamma-1)}{R e \tilde{\rho}}\left(\frac{\partial \tilde{v}}{\partial \tilde{r}}-\frac{\tilde{v}}{\tilde{r}}\right)^{2} \\
\tilde{\rho} \tilde{T}=1+\gamma M^{2} \tilde{p}
\end{gathered}
$$

Furthermore, suppose that

$$
\tilde{f}=\tilde{f_{0}}+M^{2} \tilde{f}_{1}+\ldots,
$$

where $\tilde{f}$ is any of $\tilde{u}, \tilde{v}, \tilde{\rho}, \tilde{p}$, or $\tilde{T}$. This perturbation expansion is akin to a Rayleigh-Janson expansion, applied to a viscous flow.

Retaining terms of $O(1)$ in (4.3) leads to

$$
\begin{gathered}
\frac{\partial \tilde{\rho}_{0}}{\partial \tilde{t}}+\frac{1}{\tilde{r}} \frac{\partial\left(\tilde{\rho}_{0} \tilde{u}_{0} \tilde{r}\right)}{\partial \tilde{r}}=0 \\
\tilde{\rho}_{0}\left(\frac{\partial \tilde{v}_{0}}{\partial \tilde{t}}+\tilde{u}_{0} \frac{\partial \tilde{v}_{0}}{\partial \tilde{r}}+\frac{\tilde{v}_{0} \tilde{u}_{0}}{\tilde{r}}\right)=\frac{1}{\overline{R e}} \frac{\partial}{\partial \tilde{r}}\left(\frac{1}{\tilde{r}} \frac{\partial}{\partial \tilde{r}}\left(\tilde{r} \tilde{v}_{0}\right)\right) \\
\frac{\partial}{\partial \tilde{r}}\left(\frac{1}{\tilde{r}} \frac{\partial}{\partial \tilde{r}}\left(\tilde{r} \tilde{u}_{0}\right)\right)=\frac{1}{\tilde{R e} \operatorname{Pr}} \frac{\partial}{\partial \tilde{r}}\left(\frac{1}{\tilde{r}} \frac{\partial}{\partial \tilde{r}}\left(\tilde{r} \frac{\partial \tilde{T}}{\partial \tilde{r}}\right)\right) \\
\frac{\partial \tilde{T}_{0}}{\partial \tilde{t}}+\tilde{u}_{0} \frac{\partial \tilde{T}_{0}}{\partial \tilde{r}}+(\gamma-1) \tilde{T}_{0}\left(\frac{\partial \tilde{u}_{0}}{\partial \tilde{r}}+\frac{\tilde{u}_{0}}{\tilde{r}}\right)=\frac{\gamma}{\tilde{R e} \operatorname{Pr}} \frac{1}{\tilde{r}} \frac{\partial}{\partial \tilde{r}}\left(\tilde{r} \frac{\partial \tilde{T}_{0}}{\partial \tilde{r}}\right) \\
\tilde{\rho}_{0} \tilde{T}_{0}=1
\end{gathered}
$$


For an incompressible vortex the dilatation must vanish and therefore the left-hand side of $(4.5 c)$ must vanish. Furthermore, if the dilatation vanishes,

$$
\tilde{u}_{0}=C(\tilde{l}) / \tilde{r}
$$

But the radial velocity, $\tilde{u}_{0}$ must go to zero at $\tilde{r}=0$, and therefore $\tilde{u}_{0}=0$. Then $(4.5 a)$ and $(4.5 e)$ yield

$$
\partial \tilde{\rho}_{0} / \partial \tilde{t}=0, \quad \partial \tilde{T}_{0} / \partial \tilde{t}=0 .
$$

Hence,

$$
\frac{\gamma}{\operatorname{Pr} \overline{R e} \tilde{\rho}} \frac{1}{\tilde{r}} \frac{\partial}{\partial \tilde{r}}\left(\tilde{r} \frac{\partial \tilde{T_{0}}}{\partial \tilde{r}}\right)=0
$$

If $\tilde{T}_{0}$ is to be bounded as $\tilde{r} \rightarrow 0$ and $\tilde{r} \rightarrow \infty$, then $\tilde{T}_{0}=1$, and (4.5) reduce to

$$
\begin{gathered}
\tilde{\rho}_{0}=\tilde{T}_{0}=1, \quad \tilde{u}_{0}=0, \\
\frac{\partial \tilde{v}_{0}}{\partial \tilde{t}}=\frac{1}{\tilde{R e}} \frac{\partial}{\partial \tilde{r}}\left(\frac{1}{\tilde{r}} \frac{\partial}{\partial \tilde{r}}\left(\tilde{r} \tilde{v}_{0}\right)\right), \quad \frac{\partial \tilde{p}_{0}}{\partial \tilde{r}}=\frac{\tilde{v}_{0}^{2}}{\tilde{r}} .
\end{gathered}
$$

Equations (4.9) are the well-known equations for the evolution of an incompressible, uniform-density vortex, and permit an infinite number solutions for which $\tilde{v}_{0} \rightarrow 0$ as $\tilde{r} \rightarrow 0$, and which are bounded as $\tilde{r} \rightarrow \infty$. The general solution in terms of vorticity $\left(\tilde{\omega}_{0}=(1 / \tilde{r})(\partial / \partial \tilde{r})\left(\tilde{r} \tilde{v}_{0}\right)\right)$ was found by de Neufville (1957):

$$
\tilde{\omega}_{0}=\sum_{m=0}^{\infty} C_{m} \tau^{-(m+1)} \exp (-\eta) L_{m}(\eta)
$$

where $\tau=\tilde{t} / \widetilde{R e}, \eta=\tilde{r}^{2} / 4 \tau$, and $L_{m}$ is the Laguerre polynomial of degree $m$. Mode $m=0$ has finite circulation, and infinite angular momentum. Mode $m=1$ has zero circulation, and finite total angular momentum. Higher modes have zero circulation and zero total angular momentum. The vorticity in the zeroth mode has the same sign everywhere, while in general mode $m$ has $m$ zero crossings of vorticity. The decay of an arbitrary initial distribution of vorticity can be found as a superposition of the various modes, because $(4.9 \mathrm{c})$ is linear. The constants $C_{m}$ are then determined by the initial distribution, using the orthogonality property of the Laguerre polynomials. Let the initial time be denoted by $\tau=\tau_{\mathrm{i}}$, where $\tau_{\mathrm{i}} \neq 0$. Then

$$
C_{m}=\left.\tau_{\mathrm{i}}^{m+1} \int_{0}^{\infty} \tilde{\omega}_{0}\right|_{\tau=\tau_{\mathrm{i}}} L_{m}\left(\eta_{\mathrm{i}}\right) \mathrm{d} \eta_{\mathrm{i}}
$$

The resulting tangential velocity is

$$
\tilde{v}_{0}=\frac{2 C^{0}}{\tilde{r}}(1-\exp (-\eta))+\sum_{m=1}^{\infty} \frac{2 C_{m} \tau^{-m}}{\tilde{r}} \exp (-\eta)\left(L_{m-1}(\eta)-L_{m}(\eta)\right) \text {. }
$$

And $\tilde{p}_{0}$ can be found:

$$
\tilde{p}_{0}=-\int_{\tilde{r}}^{\infty} \frac{\tilde{v}_{0}^{2}}{\tilde{r}} \mathrm{~d} \tilde{r}
$$

The shapes of the first few modes of the vorticity, tangential velocity and pressure are shown in figure 7. $\left(C_{m}=1\right.$, and $\tau=1$ in figure 7.) Mode 1 corresponds to the solution due to Oseen (1912), and mode 2 corresponds to the solution due to Taylor (1918), and also studied by Long (1961), Sibulkin (1961), Morton (1969), and Uberoi (1979). 

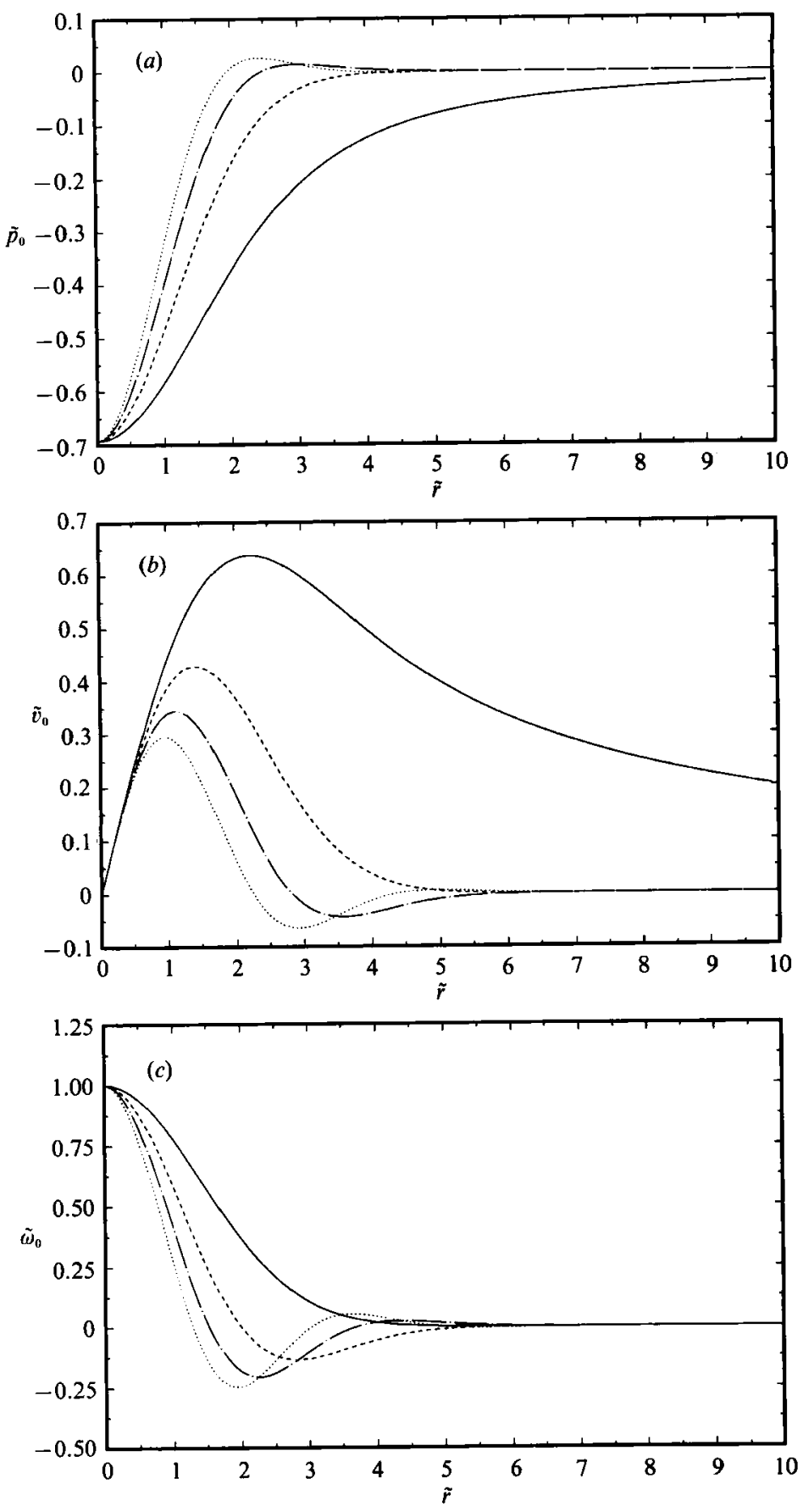

Figure 7. First four modes of the analytical solution for an incompressible vortex, $(a)$ pressure, (b) tangential velocity, $(c)$ vorticity:,$- m=0 ;----, m=1 ;-\cdots, m=2 ; \cdots \cdots-\cdot, m=3$. 
Terms of $O\left(M^{2}\right)$ in (4.3) are given by

$$
\begin{gathered}
\frac{\partial \tilde{\rho}_{1}}{\partial \tilde{t}}+\frac{1}{\tilde{r}} \frac{\partial\left(\tilde{r} \tilde{u}_{1}\right)}{\partial \tilde{r}}=0 \\
\frac{\partial \tilde{v}_{1}}{\partial \tilde{t}}+\tilde{\rho}_{1} \frac{\partial \tilde{v}_{0}}{\partial \tilde{t}}+\tilde{u}_{1}\left(\frac{\partial \tilde{v}_{0}}{\partial \tilde{r}}+\frac{\tilde{v}_{0}}{\tilde{r}}\right)=\frac{1}{\overline{R e}} \frac{\partial}{\partial \tilde{r}}\left(\frac{1}{\tilde{r}} \frac{\partial}{\partial \tilde{r}}\left(\tilde{r} \tilde{v}_{1}\right)\right) \\
\frac{\partial}{\partial \tilde{r}}\left(\frac{1}{\tilde{r}} \frac{\partial}{\partial \tilde{r}}\left(\tilde{r} \tilde{u}_{1}\right)\right)=\frac{-2 \tilde{v}_{0}}{\overline{R e} \tilde{r}} \frac{\partial}{\partial \tilde{r}}\left(\frac{1}{\tilde{r}} \frac{\partial}{\partial \tilde{r}}\left(\tilde{r} \tilde{v}_{0}\right)\right)+\frac{1}{\overline{R e} \operatorname{Pr}} \frac{\partial}{\partial \tilde{r}}\left(\frac{1}{\tilde{\mathbf{r}}} \frac{\partial}{\partial \tilde{r}}\left(\tilde{r} \frac{\partial \tilde{T}_{1}}{\partial \tilde{r}}\right)\right)+\frac{(\gamma-1)}{\overline{R e}} \frac{\partial}{\partial \tilde{r}}\left(\frac{\partial \tilde{v}_{0}}{\partial \tilde{r}}-\frac{\tilde{v}_{0}}{\tilde{r}}\right)^{2}, \\
\frac{\partial \tilde{T}_{1}}{\partial \tilde{t}}+(\gamma-1)\left(\frac{\partial \tilde{u}_{1}}{\partial \tilde{r}}+\frac{\tilde{u}_{1}}{\tilde{r}}\right)=\frac{\gamma}{\overline{R e} \operatorname{Pr}} \frac{1}{\tilde{r}} \frac{\partial}{\partial \tilde{r}}\left(\tilde{r} \frac{\partial \tilde{T}}{\partial \tilde{r}}\right)+\frac{\gamma(\gamma-1)}{\overline{R e}}\left(\frac{\partial \tilde{v}_{0}}{\partial \tilde{r}}-\frac{\tilde{v}_{0}}{\tilde{r}}\right)^{2}, \\
\tilde{\rho}_{1}+\tilde{T}_{1}=\gamma \tilde{p}_{0}, \quad \frac{\partial \tilde{p}_{1}}{\partial \tilde{r}}=\frac{\tilde{\rho}_{1} \tilde{v}_{0}^{2}}{\tilde{r}}+\frac{2 \tilde{v}_{1} \tilde{v}_{0}}{\tilde{r}} .
\end{gathered}
$$

Equations (4.14) are now sufficiently simple to yield analytical solutions. Integrating $(4.14 c)$ twice gives the radial velocity, $\tilde{u}_{1}$, in terms of the density perturbation and the $O(1)$ solutions:

$$
\overline{R e} \tilde{u}_{1}=\frac{1}{\tilde{r}} \int_{0}^{\tilde{r}} \tilde{r}\left(\int_{\tilde{r}}^{\infty} \frac{2 \tilde{v}_{0}}{\tilde{r}} \frac{\partial \omega_{0}}{\partial \tilde{r}} \mathrm{~d} \tilde{r}\right) \mathrm{d} \tilde{r}+\frac{(\gamma-1)}{\tilde{r}} \int_{0}^{\tilde{r}} \tilde{r}\left(\frac{\partial \tilde{v}_{0}}{\partial \tilde{r}}-\frac{\tilde{v}_{0}}{\tilde{r}}\right)^{2} \mathrm{~d} \tilde{r}+\frac{\gamma}{\operatorname{Pr}}\left(\frac{\tilde{v}_{0}^{2}}{\tilde{r}}\right)-\frac{1}{\operatorname{Pr}} \frac{\partial \tilde{\rho}_{1}}{\partial \tilde{r}},
$$

where $(4.14 e)$ and the requirement that the radial velocity vanish as $\tilde{r} \rightarrow 0$ and $\tilde{r} \rightarrow$ $\infty$ have been used.

Rearranging $(4.14 a)$ and substituting for $\tilde{u}_{1}$ from $(4.15)$, where $(4.14 e)$ and $(4.9 d)$ have been used, gives

$$
\begin{aligned}
\frac{\partial \tilde{\rho}_{1}}{\partial \tau}-\frac{1}{\operatorname{Pr}} \frac{1}{\tilde{r}} \frac{\partial}{\partial \tilde{r}}\left(\tilde{r} \frac{\partial \tilde{\rho}_{1}}{\partial \tilde{r}}\right)= & \frac{-\gamma}{\operatorname{Pr}} \frac{1}{\tilde{r}} \frac{\partial}{\partial \tilde{r}}\left(\tilde{v}_{0}^{2}\right) \\
& +\int_{\tilde{r}}^{\infty} \frac{-2 \tilde{v}_{0}}{\tilde{r}} \frac{\partial}{\partial \tilde{r}}\left(\frac{1}{\tilde{r}} \frac{\partial}{\partial \tilde{r}}\left(\tilde{r} \tilde{v}_{0}\right)\right) \mathrm{d} \tilde{r}-(\gamma-1)\left(\frac{\partial \tilde{v}_{0}}{\partial \tilde{r}}-\frac{\tilde{v}_{0}}{\tilde{r}}\right)^{2}
\end{aligned}
$$

Finally, $(4.14 b)$ is differentiated to give an equation for the vorticity perturbation in terms of the density perturbation, the radial velocity, and the $O(1)$ vorticity:

$$
\frac{\partial \tilde{\omega}_{1}}{\partial \tau}-\frac{1}{\tilde{r}} \frac{\partial}{\partial \tilde{r}}\left(\tilde{r} \frac{\partial \tilde{\omega}_{1}}{\partial \tilde{r}}\right)=\tilde{\rho}_{1} \frac{\partial \tilde{\omega}_{0}}{\partial \tau}-\tilde{\omega}_{0} \frac{\partial \tilde{\rho}_{1}}{\partial \tau}+\left(\frac{\partial \tilde{\rho}_{1}}{\partial \tilde{r}}+\widetilde{R e} \tilde{u}_{1}\right) \frac{\partial \tilde{\omega}_{0}}{\partial \tilde{r}}
$$

Initial and boundary conditions are needed for both $\tilde{\rho}_{1}$ and $\tilde{\omega}_{1}$. It is required that $\tilde{\rho}_{1}$ decay and $\tilde{\omega}_{1} \rightarrow 0$ exponentially fast as $\tilde{r} \rightarrow \infty$. Owing to the cylindrical geometry, the slope of $\tilde{\rho}_{1}$ and $\tilde{\omega}_{1}$ must go to zero as $\tilde{r}$ goes to zero. Initial conditions are also expanded in terms of Mach number as

$$
\begin{aligned}
& \tilde{\rho}\left(\tilde{r}, \tau_{\mathrm{i}}\right)=1+M^{2} \tilde{\rho}_{1}\left(\tilde{r}, \tau_{\mathrm{i}}\right)+\ldots \\
& \tilde{\omega}\left(\tilde{r}, \tau_{\mathrm{i}}\right)=\tilde{\omega}_{\mathbf{0}}\left(\tilde{r}, \tau_{\mathrm{i}}\right)+M^{2} \tilde{\omega}_{1}\left(\tilde{r}, \tau_{\mathrm{i}}\right)+\ldots
\end{aligned}
$$

We note that initial conditions such as (4.18) should be determined by matching a solution to (2.1) for small times (inner solution) from an arbitrary initial condition, to the solutions to (4.3), the outer solution. Such an inner solution which resolves the 
acoustic transient, varies on two distinct spatial scales - the near vortex region of scale $L$, and a far-field region of scale $a_{\infty} t$. This asymptotic matching procedure is not attempted here, but useful information can be gained without it. Assume that the initial conditions for the outer solution are known, then solutions to (4.14) can be found in terms of the arbitrary functions $\tilde{\rho}_{1}\left(\tilde{r}, \tau_{1}\right), \tilde{\omega}_{0}\left(\tilde{r}, \tau_{1}\right)$, and $\tilde{\omega}_{1}\left(\tilde{r}, \tau_{1}\right)$. In the next section, the behaviour of the solution for particular initial conditions will be examined.

\subsection{Low-Mach-number corrections to the Oseen vortex}

We now restrict our attention to the case where the $(O(1))$ vorticity, $\tilde{\omega}_{0}$, is given by

$$
\left.\tilde{\omega}_{0}\right|_{\tau-\tau_{1}}=\frac{\Gamma}{4 \pi v_{\mathrm{m}} L \tau} \exp (-\eta)
$$

which corresponds to the incompressible solution found by Oseen (1912). $\Gamma$ is the circulation of the vortex as defined by $\Gamma=\int_{A} \omega \mathrm{d} A$. Hence the constants in (4.10) are given by

and therefore,

$$
C_{0}=\frac{\Gamma}{4 \pi v_{\mathrm{m}} L}, \quad C_{m}=0, \quad m=1,2, \ldots,
$$

$$
\begin{aligned}
& \tilde{v}_{0}=\frac{\Gamma}{2 \pi v_{\mathrm{m}} L \tilde{r}}(1-\exp (-\eta)) \\
& \tilde{p}_{0}=-\frac{\Gamma^{2}}{16 \pi^{2} v_{\mathrm{m}}^{2} L^{2} \tau}\left(\frac{1}{2 \eta}(1-2 \exp (-\eta)+\exp (-2 \eta))+\operatorname{Ei}(-2 \eta)-\operatorname{Ei}(-\eta)\right)
\end{aligned}
$$

where Ei is the exponential integral, e.g. Abramowitz \& Stegun (1972).

If $v_{\mathrm{m}}$ is chosen to be the maximum tangential velocity at $\tau=\tau_{1}$, and $L$ to be the radius at the point where the velocity is maximum at $\tau=\tau_{1}, C_{0}$ and $\tau_{1}$ are fixed and

$$
C_{0} \approx 0.699, \quad \tau_{\mathrm{i}} \approx 0.197 \text {. }
$$

If (4.19) and (4.21 $a)$ are substituted into the right-hand side of (4.16) the result is

$$
\begin{aligned}
\frac{\partial \tilde{\rho}_{1}}{\partial \tau}- & \frac{1}{\operatorname{Pr}} \frac{1}{\tilde{r}} \frac{\partial}{\partial \tilde{r}}\left(\tilde{r} \frac{\partial \tilde{\rho}_{1}}{\partial \tilde{r}}\right)=\frac{C_{0}^{2}}{\tau^{2}}[\operatorname{Ei}(-2 \eta)-\operatorname{Ei}(-\eta)-(\gamma-1) \exp (-2 \eta) \\
+ & \frac{1}{\eta}\left(\frac{-\gamma}{\operatorname{Pr}}+2 \gamma-2\right)(\exp (-\eta)-\exp (-2 \eta)) \\
& \left.+\frac{1}{\eta^{2}}\left(\frac{\gamma}{2 P r}-\gamma+1\right)(1-2 \exp (-\eta)+\exp (-2 \eta))\right] .
\end{aligned}
$$

$\tilde{\rho}_{1}$ is now split into a homogeneous and particular solution to (4.23), i.e.

$$
\tilde{\rho}_{\mathbf{1}}(\tilde{r}, \tau)=\tilde{\rho}_{\mathbf{1}}^{\mathrm{h}}(\tilde{r}, \tau)+\tilde{\rho}_{1}^{\mathrm{p}}(\tilde{r}, \tau)
$$

A particular solution which satisfies (4.23) and decays as $\tilde{r} \rightarrow \infty$ has been found (the details have been omitted). It is

$$
\begin{aligned}
\tilde{\rho}_{1}^{\mathrm{p}}=\frac{C_{0}^{2} \operatorname{Pr}}{\tau}[\exp ( & -\operatorname{Pr} \eta)\left(c_{1} \operatorname{Ei}(\operatorname{Pr} \eta)+c_{2} \operatorname{Ei}((\operatorname{Pr}-1) \eta)+c_{3} \operatorname{Ei}((\operatorname{Pr}-2) \eta)\right) \\
& \left.+\frac{c_{4}}{\eta}(1-2 \exp (-\eta)+\exp (-2 \eta))+\frac{1}{\operatorname{Pr}}(\operatorname{Ei}(-\eta)-\operatorname{Ei}(-2 \eta))\right],
\end{aligned}
$$


where

$$
\begin{gathered}
c_{1}=-(\gamma-1)(\operatorname{Pr}-1), \quad c_{2}=\frac{1}{\operatorname{Pr}}(\gamma-1)(\operatorname{Pr}-1)(2 \operatorname{Pr}-1), \quad(4.26 a, b) \\
c_{3}=-\frac{1}{\operatorname{Pr}}(\gamma-1)(\operatorname{Pr}-1)^{2}, \quad c_{4}=(\gamma-1)-\frac{\gamma}{2 \operatorname{Pr}} .
\end{gathered}
$$

The homogeneous problem is now

$$
\frac{\partial \tilde{\rho}_{1}^{\mathrm{h}}}{\partial \tau}-\frac{1}{\operatorname{Pr}} \frac{1}{\tilde{r}} \frac{\partial}{\partial \tilde{r}}\left(\tilde{r} \frac{\partial \tilde{\rho}_{1}^{\mathrm{h}}}{\partial \tilde{r}}\right)=0
$$

and the initial condition is now

$$
\tilde{\rho}_{1}^{\mathrm{h}}\left(\tilde{r}, \tau_{\mathrm{i}}\right)=\tilde{\rho}_{1}\left(\tilde{r}, \tau_{\mathrm{i}}\right)-\tilde{\rho}_{1}^{\mathrm{p}}\left(\tilde{r}, \tau_{\mathrm{i}}\right),
$$

and $\tilde{\rho}_{1}^{\text {h }}$ must decay as $\tilde{r} \rightarrow \infty$. The general solution to (4.27) is well known and its solution can be written as

$$
\tilde{\rho}_{1}^{\mathrm{h}}(\tilde{r}, \tau)=2 \pi \int_{0}^{\infty} \bar{\rho}_{1}\left(q, \tau_{\mathrm{i}}\right) \exp \left(\frac{-4 \pi^{2} q^{2}\left(\tau-\tau_{\mathrm{i}}\right)}{\operatorname{Pr}}\right) J_{0}(2 \pi q \tilde{r}) q \mathrm{~d} q
$$

where $\bar{\rho}_{1}\left(q, \tau_{1}\right)$ is the Hankel transform of the initial condition,

$$
\bar{\rho}_{1}\left(q, \tau_{\mathrm{i}}\right)=2 \pi \int_{0}^{\infty} \tilde{\rho}_{1}^{\mathrm{h}}\left(x, \tau_{\mathrm{i}}\right) J_{0}(2 \pi q x) x \mathrm{~d} x .
$$

Combining (4.29) and (4.30) and performing one of the integrations gives

$$
\tilde{\rho}_{1}^{\mathrm{h}}(\tilde{r}, \tau)=\frac{\operatorname{Pr}}{2\left(\tau-\tau_{\mathrm{i}}\right)} \exp \left(\frac{-\tilde{r}^{2} \operatorname{Pr}}{4\left(\tau-\tau_{\mathrm{i}}\right)}\right) \int_{0}^{\infty} \tilde{\rho}_{1}^{\mathrm{h}}\left(x, \tau_{1}\right) \exp \left(\frac{-x^{2} \operatorname{Pr}}{4\left(\tau-\tau_{\mathrm{j}}\right)}\right) I_{0}\left(\frac{\operatorname{Pr} \tilde{r} x}{2\left(\tau-\tau_{\mathrm{i}}\right)}\right) x \mathrm{~d} x
$$

where $I_{0}$ is the modified Bessel function.

The solution for $\tilde{\rho}_{1}$ can now be substituted into the right-hand side of (4.15), and the radial velocity $\tilde{u}_{1}$ is determined. Likewise, the temperature perturbation $\tilde{T}_{1}$ can be determined from (4.14e). The right-hand side of (4.17) is also known in terms of the solutions for $\tilde{\rho}_{1}, \tilde{u}_{1}$ and the $O(1)$ solutions, and the vorticity perturbation $\tilde{\omega}_{1}$ can be found by solving the (4.17) subject to the boundary and initial conditions.

We proceed by taking the Hankel transform of (4.17):

where

$$
\mathrm{d} \bar{\omega}_{1} / \mathrm{d} \tau(q, \tau)+4 \pi q^{2} \bar{\omega}_{1}(q, \tau)=\bar{Q}(q, \tau),
$$

is the Hankel transform of the vorticity perturbation, and

$$
\bar{Q}(q, \tau)=2 \pi \int_{0}^{\infty}\left[\tilde{\rho}_{1} \frac{\partial \tilde{\omega}_{0}}{\partial \tau}-\tilde{\omega}_{0} \frac{\partial \tilde{\rho}_{1}}{\partial \tau}+\left(\frac{\partial \tilde{\rho}_{1}}{\partial \tilde{r}}+\tilde{u}_{1}\right) \frac{\partial \tilde{w}_{0}}{\partial \tilde{r}}\right] J_{0}(2 \pi \tilde{r} q) \tilde{r} \mathrm{~d} \tilde{r}
$$

is the Hankel transform of the right-hand side of (4.17). Solving the ODE (4.32), gives

$$
\bar{\omega}_{1}(q, \tau)=\exp \left(-4 \pi q^{2} \tau\right) \int_{\tau_{\mathrm{i}}}^{\tau} \exp \left(4 \pi q^{2} \tau^{\prime}\right) \bar{Q}\left(q, \tau^{\prime}\right) \mathrm{d} \tau^{\prime}+\bar{\omega}_{1}\left(q, \tau_{\mathrm{i}}\right) \exp \left(-4 \pi q^{2}\left(\tau-\tau_{\mathrm{i}}\right)\right)
$$



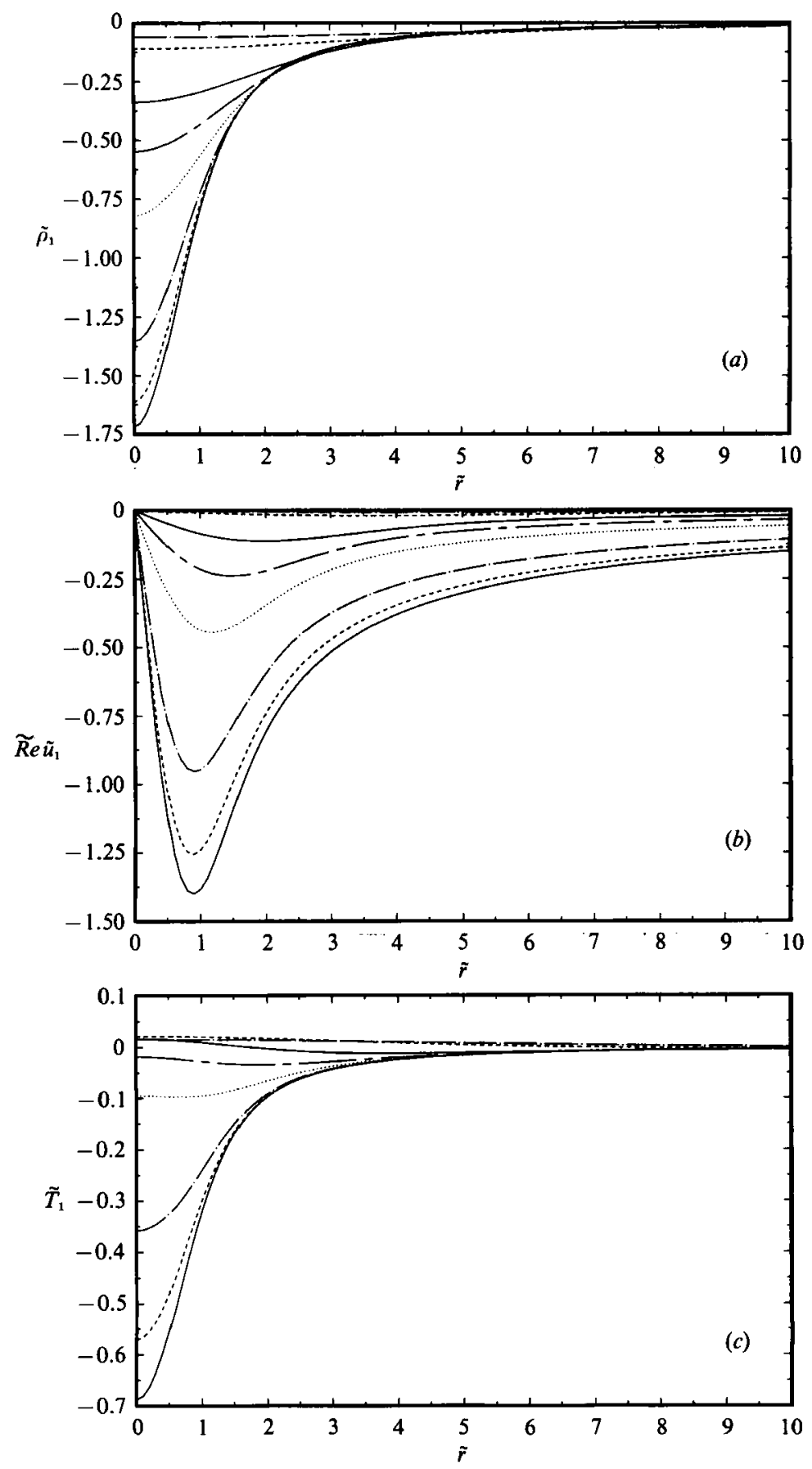

Figure 8 $(a-c)$. For caption see p. 65.

where $\bar{\omega}\left(q, \tau_{1}\right)$ is the Hankel transform of the initial condition $\tilde{\omega}_{1}\left(\tilde{r}, \tau_{1}\right)$. Finally, the solution for the vorticity perturbation is given by the inverse Hankel transform of (4.35):

$$
\tilde{\omega}_{1}(\tilde{r}, \tau)=2 \pi \int_{0}^{\infty} \bar{\omega}_{1}(q, \tau) J_{0}(2 \pi \tilde{r} q) q \mathrm{~d} q .
$$



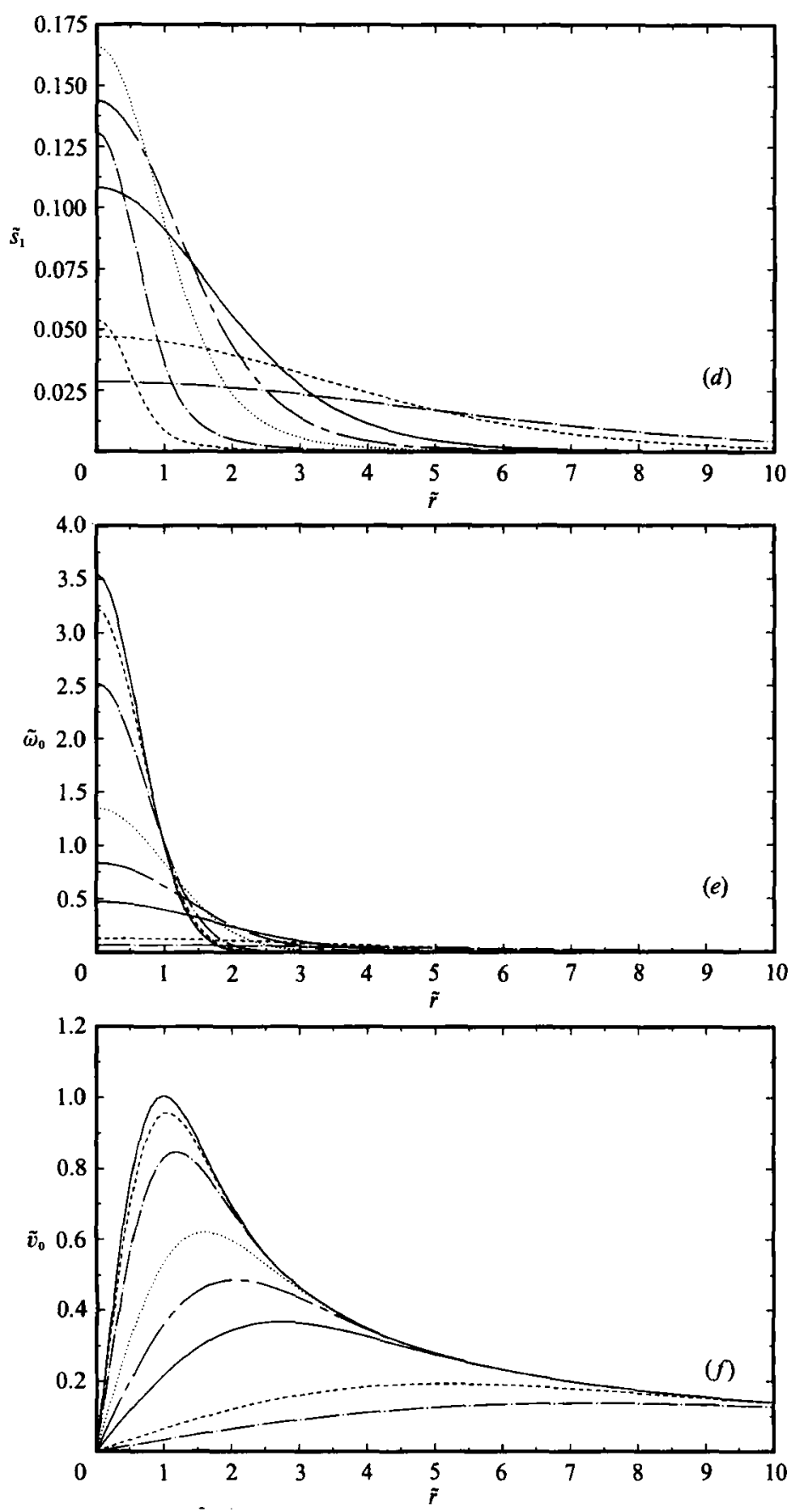

Figure $8(d-f)$. For caption see facing page.

The quadratures in (4.31) and (4.36) can be performed given the initial conditions for the density and vorticity perturbations respectively. In $\$ \$ 4.2$ and 4.3 , solutions are given for the density, radial velocity, and temperature perturbations for two particular initial conditions - a vortex which is initially homentropic, and a vortex which initially has a uniform density. 


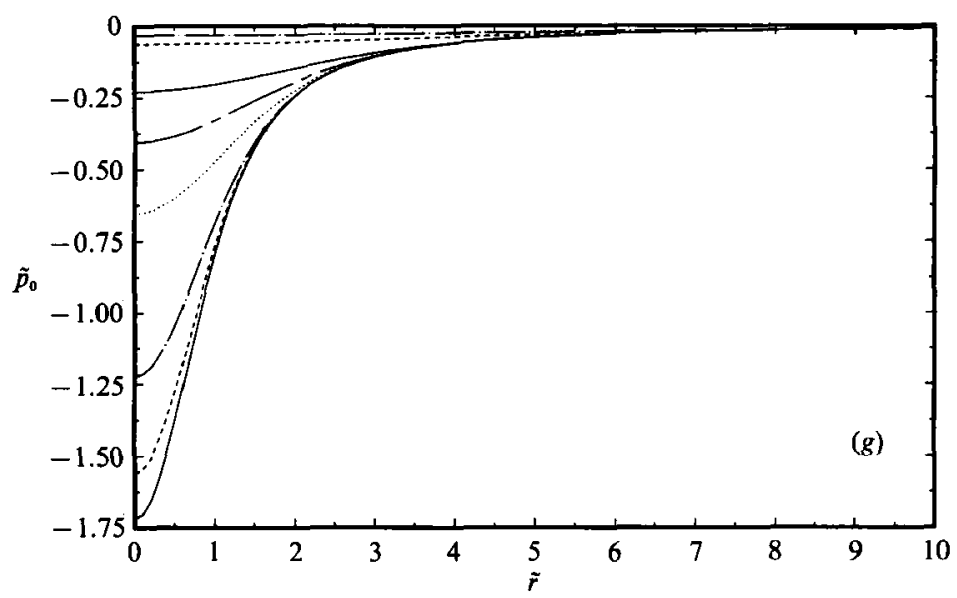

Figure 8. Evolution of $(a)$ density perturbation, $(b)$ radial velocity perturbation, $(c)$ temperature perturbation, $(d)$ entropy perturbation, $(e)$ vorticity, $(f)$ tangential velocity, and $(g)$ pressure : $\tau=\tau_{1} ;-\cdots, \tau=0.217 ;-.-, \tau=0.277 ; \cdots,-\cdots, \tau=0.517 ;-\square, \tau=0.837 ;-, \tau=1.477$; ,$---- \tau=5.317 ;-.-, \tau=10.437$.

\subsection{Evolution of an initially homentropic vortex}

The entropy distribution to $O\left(M^{2}\right)$ in the vortex is determined from the incompressible pressure and the $O\left(M^{2}\right)$ density by

$$
\left.\begin{array}{rl}
\tilde{s}=\frac{s-s_{\infty}}{c_{p}} & =1 / \gamma \ln \left(p / p_{\infty}\right)-\ln \left(\rho / \rho_{\infty}\right) \\
& =M^{2}\left(\tilde{p}_{0}-\tilde{\rho}_{1}\right)+O\left(M^{4}\right) . \\
\tilde{s}_{1} & =\tilde{p}_{0}-\tilde{\rho}_{1} .
\end{array}\right\}
$$

Therefore the initial condition for $\tilde{\rho}_{1}$ for an initially homentropic vortex is

$$
\begin{aligned}
\tilde{\rho}_{1}\left(\tilde{r}, \tau_{\mathrm{i}}\right) & =\tilde{p}_{0}\left(\tilde{r}, \tau_{\mathrm{i}}\right) \\
& =-\frac{C_{0}^{2}}{\tau_{\mathrm{i}}}\left(\frac{1}{2 \eta_{\mathrm{i}}}\left(1-2 \exp \left(-\eta_{\mathrm{i}}\right)+\exp \left(-2 \eta_{\mathrm{i}}\right)\right)+\operatorname{Ei}\left(-2 \eta_{\mathrm{i}}\right)-\mathrm{Ei}\left(-\eta_{\mathrm{i}}\right)\right) .
\end{aligned}
$$

The particular solution (4.25) at $\tau=\tau_{\mathrm{i}}$ is subtracted from (4.38) and the result, $\tilde{\rho}_{1}^{\mathrm{h}}\left(\tilde{r}, \tau_{1}\right)$, is substituted into (4.31). The quadrature in $(4.31)$ is performed numerically by first mapping the infinite domain to $(-1,1)$ and using Gaussian quadrature with as many as 500 quadrature points depending on the values of $\tilde{r}$ and $\tau$ in (4.31). $\operatorname{Pr}$ and $\gamma$ are taken to be $\frac{3}{4}$ and 1.4 respectively.

The radial velocity $\tilde{u}_{1}$ is determined by substituting $(4.31),(4.25),(4.21 a)$ and (4.19) into the right-hand side of $(4.16)$. The quadratures are again performed numerically. The temperature perturbation $\tilde{T}_{1}$ is found by substituting the expressions for $\tilde{\rho}_{1}$ and $\tilde{p}_{0}$ into $(4.14 e)$.

The resulting distributions of $\tilde{\rho}_{1}, \tilde{u}_{1}, \tilde{T}_{1}$ and $\tilde{s}_{1}$ are shown in figure 8 versus $\tilde{r}$ at various times. In order to show all phases of the vortex evolution on one plot, the time increment in the figure is not uniform. For reference, the distributions of the first-order variables $\tilde{\omega}_{0}, \tilde{v}_{0}$, and $\tilde{p}_{0}$ are also shown in figure 8 .

In the incompressible Oseen solution, viscous effects are limited to a region in the 


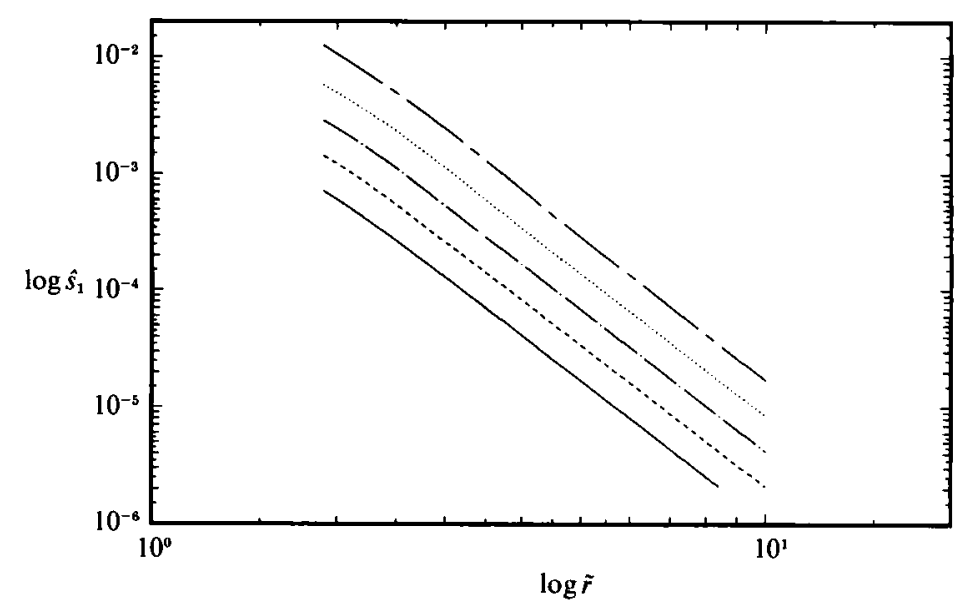

Figure 9. Evolution of entropy perturbation:,$- \tau=0.207 ;----, \tau=0.217$; $-\cdot-, \tau=0.237 ; \cdots \cdots, \tau=0.277 ;--\longrightarrow, \tau=0.357$.

core of the vortex. The viscous region spreads outward like $\tilde{r} \sim \tau^{\frac{1}{2}}$. The pressure (figure $8 g$ ) is initially lower than ambient and decays to the ambient conditions on the viscous timescale. For $\tilde{r} \gg \tau^{\frac{1}{2}}$ the pressure does not change in time, and decays as $1 / \tilde{r}^{2}$. For the initially homentropic vortex, the $O\left(M^{2}\right)$ density correction (figure $8 a$ ) is initially equal to the pressure. The density decays in time in a manner similar to the incompressible pressure, but at a slightly different rate such that the entropy in the core (figure $8 d$ ) does not remain uniform. Far from the core the density changes in time to $O\left(1 / \tilde{r}^{4}\right)$, and therefore the entropy also changes in time to $O\left(1 / \tilde{r}^{4}\right)$. This is due to the viscous dissipation of energy and heat conduction which create entropy even far from the core of the vortex. To $O\left(M^{2}\right)$ the entropy change is given by

$$
\frac{\partial \tilde{s}_{1}}{\partial \tau}=(\gamma-1)\left(\frac{\partial \tilde{v}_{0}}{\partial \tilde{r}}-\frac{\tilde{v}_{0}}{\tilde{r}}\right)^{2}+\frac{1}{\operatorname{Pr}} \frac{1}{\tilde{r}} \frac{\partial}{\partial \tilde{r}}\left(\tilde{r} \frac{\partial \tilde{T}_{1}}{\partial \tilde{r}}\right)
$$

Both terms on the right-hand side of (4.39) decay like $1 / \widetilde{r}^{4}$ far from the core, which gives the $1 / \tilde{r}^{4}$ entropy decay shown in figure 9 where $\log \tilde{s}_{1}$ has been plotted versus $\log \tilde{r}$; the slope of the curves is -4 away from the core.

Inside the vortex core, the entropy first increases with time, but eventually peaks and begins to decay. This can be understood by examining the balance of terms on the right-hand side of (4.39). The viscous dissipation term is always positive, while the heat conduction term can be positive or negative. For the initial temperature profile (figure $8 c$ ), the heat conduction term is positive in the core and negative away from it. Away from the core the viscous dissipation term dominates the balance, and the entropy increases everywhere initially. Eventually, as the density and pressure decay at different rates, the temperature becomes greater than ambient in the core, and the sign of the heat conduction term in (4.39) is reversed, and therefore the entropy in the core begins to decrease in time. Thus we may think of the evolution of the vortex to be in two phases. First, the core region relaxes from the 'nonequilibrium' initial condition, so that the entropy is larger than ambient. Then the entropy in the core decays, so that the entropy returns to its ambient value as the vortex dies out. At this stage, at a given observation point outside the vortex core, the entropy is always increasing owing to the dominant viscous dissipation term in (4.39), but eventually the viscous core (which spreads as $\tau^{\frac{1}{2}}$ ) reaches the observation 

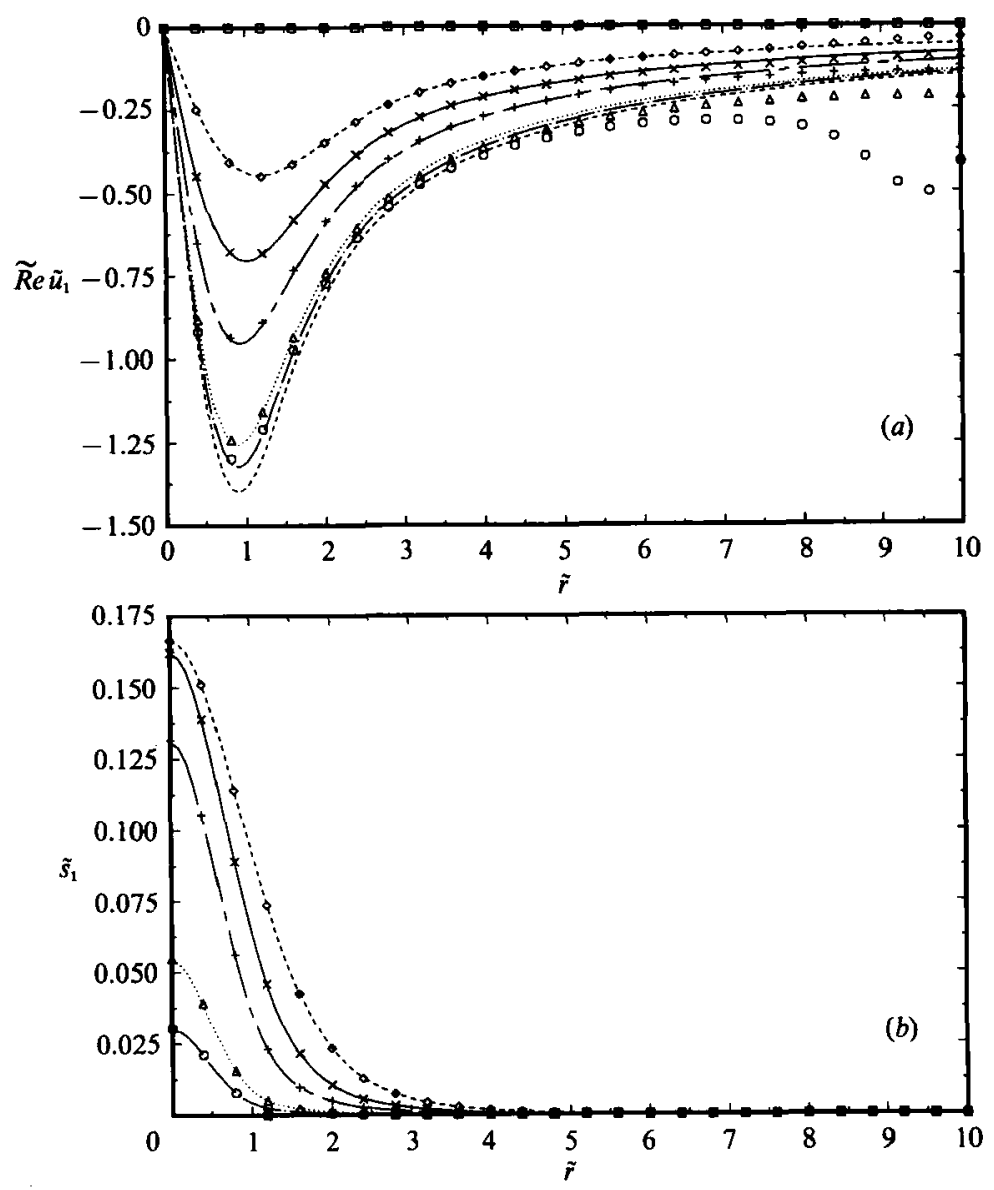

Figure 10. Evolution of $(a)$ radial velocity, $(b)$ entropy. Lines from analytical solution:---$\tau=\tau_{1} ; \cdot-, \tau=0.207 ; \cdots . .-., \tau=0.217 ;--\cdots, \tau=0.277 ;-, \tau=0.357 ;----, \tau=0.512$. Symbols from numerical solution: $\square, \tau=\tau_{1} ; O, \tau=0.207 ; \triangle, \tau=0.217 ;+, \tau=0.277 ; \times, \tau=$ $0.357 ; \diamond, \tau=0.512$.

point and the entropy decays. Note that the second phase, where the entropy is decaying in the core, occurs after long times when the vorticity at $\tilde{r}=0$ has decayed to about $25 \%$ of its initial value.

The induced radial velocity (figure $8 b$ ) is negative and has a profile which is similar to the tangential velocity. The magnitude of the radial velocity reaches a maximum near the edge of the vortex core, which spreads outward at a rate proportional to $\tau^{\frac{1}{2}}$. Far from the core the radial velocity decays as $1 / \tilde{r}$, but, unlike the tangential velocity, also decays in time like $1 / \tau$.

We note that the preceding arguments for the far field are valid far from the vortex core, but not so far as the acoustic disturbance which propagates away from the vortex at the sonic velocity, i.e. $(v t)^{\frac{1}{2}} \ll r \ll a_{\infty} t$. It is of interest, at this point, to compare the solutions given above with a numerical solution to the full equations (2.1) which resolves the acoustic disturbance. A numerical solution to the full equations is found for $M=0.125$ and $\overline{R e}=125$ (the details of our numerical solution scheme were given in $\S 3.1$ ). The initial condition is homentropic, and the velocity, and pressure given by $(4.21)$ are used as initial conditions. The radial velocity is 
initially set to zero. In figure 10 the radial velocity $\tilde{v}_{1}$ and the entropy $\tilde{s}_{1}$ from the numerical solution are compared with the analytical solution for several times. The agreement in the entropy profiles is good, including the $1 / \tilde{r}^{4}$ decay of entropy away from the vortex core. The radial velocity from the numerical solution at $\tau=0.207$ shows the acoustic disturbance created at $\tau=\tau_{\mathrm{i}}$ which has travelled away from the core at the sonic velocity. Behind the disturbance $(\tau>0.207)$, the agreement between the numerical and analytical solutions is again good. The agreement between the numerical and analytical solutions for $\tilde{v}_{0}, \tilde{p}_{0}, \tilde{T}_{1}$, and $\tilde{\rho}_{1}$ is also good.

\subsection{Evolution of an initially uniform-density vortex}

For a vortex which initially has a uniform density,

$$
\tilde{\rho}_{1}\left(\tilde{r}, \tau_{1}\right)=0 .
$$

Following the same procedure as in $\S 4.2$, we solve for the evolution of $\tilde{\rho}_{1}, \tilde{u}_{1}$, and $\tilde{T}_{1}$. The resulting distributions of $\tilde{\rho}_{1}, \tilde{u}_{1}, \tilde{T}_{1}$ and $\tilde{s}_{1}$ are shown in figure 11 versus $\tilde{r}$ at various times (the first-order variables, $\tilde{p}_{0}, \tilde{v}_{0}$, and $\tilde{\omega}_{0}$, are identical to those shown in figure 8).

Since the density is initially uniform, the entropy perturbation (figure $11 d$ ) is initially equal to the pressure. The entropy in the core decays to ambient on the viscous timescale, in the same manner as the incompressible pressure. Outside the vortex core, viscous dissipation and heat conduction again cause the entropy to change with time to $O\left(1 / \tilde{r}^{4}\right)$, as in the initially homentropic vortex. This causes the density (figure $11 a$ ) away from the vortex core to increase as $O\left(1 / \tilde{r}^{4}\right)$. Near the core region, the density profile is more complicated. At early times, the density becomes lower than ambient inside the core, and higher than ambient further away. As time increases, the density 'well' fills up and the density becomes larger than ambient everywhere in the vortex. Eventually, the density in the core of the vortex reaches a maximum, and begins to decay. Outside the spreading vortex core, the density is always increasing in time at a rate proportional to $1 / \tilde{r}^{4}$. Again, this phase of the evolution occurs after long times, when the centreline vorticity has decays to $25 \%$ of its initial value. Note that the density is very small ( 20 fold smaller) for all times compared to the density for the homentropic initial condition.

The radial velocity (figure $11 b$ ) is initially positive in the core, and negative outside it. As the vortex core spreads, the radial velocity near its edge changes sign to positive values, which is consistent with the eventual decrease of the density in the core, as the vortex decays.

\subsection{Discussion}

We have determined the evolution of the $O\left(M^{2}\right)$ corrections to the distributions of the density, temperature, entropy, and radial velocity for the Oseen vortex, for two specific initial conditions, i.e. uniform entropy and uniform density. The framework of the analysis, however, is sufficiently general to compute the evolution from any arbitrary initial condition, subject to the constraints of low Mach number, and the neglect of acoustic transients.

Furthermore, the analysis can be easily generalized to $O(1)$ two-dimensional axisymmetric vortex flows other than the Oseen vortex. From (4.10) any of these flows can be expressed as a superposition of an infinite number of vorticity modes. The Oseen vortex corresponds to the first mode, and is the only mode which has a non-zero circulation and hence an algebraically decaying tangential velocity in the far field. All other modes have velocity profiles which decay exponentially fast in the far field. Hence the particular solution to (4.16) for other $O(1)$ flows must also decay 

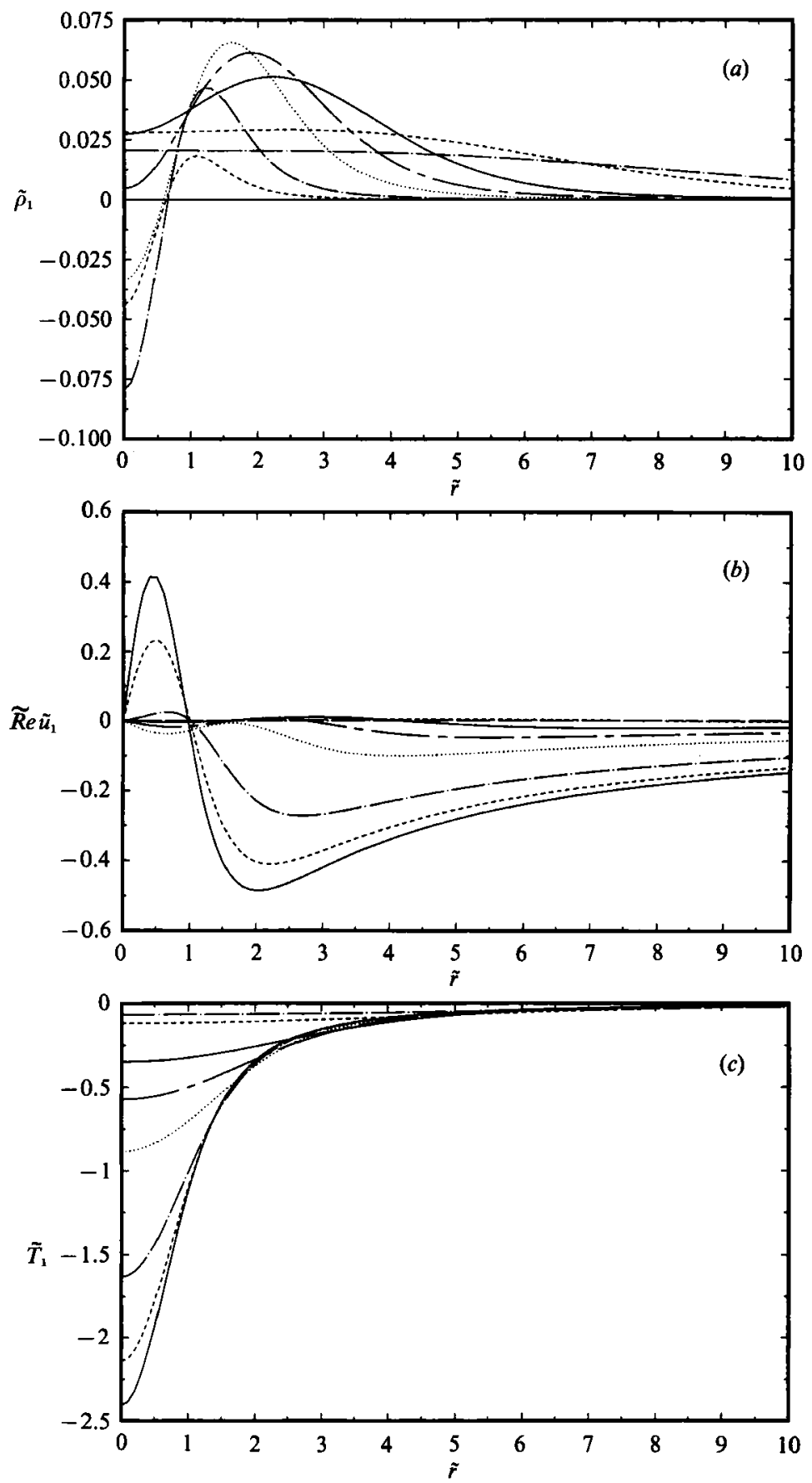

Figure $11(a-c)$. For caption see facing page.

exponentially fast. Therefore homogeneous solutions to (4.16) and the particular solution for the $O(1)$ Oseen vortex are the only axisymmetric solutions to (4.16) which will decay algebraically in the far field. Physically, the $1 / \tilde{r}$ tangential velocity of the Oseen vortex produces viscous effects (i.e. viscous dissipation and heat conduction) which are not limited to the vortex core. As we discuss later in this 


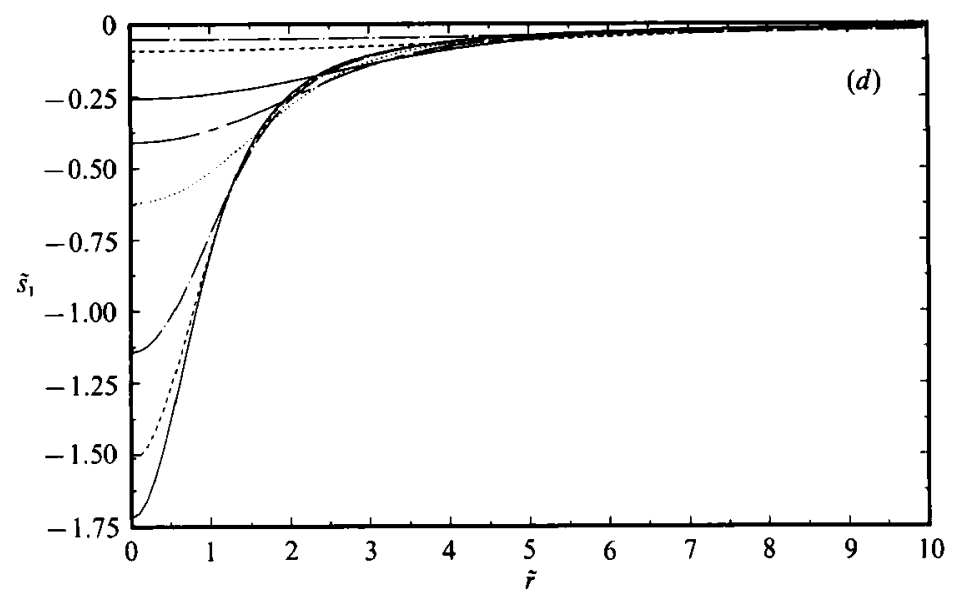

Figure 11. Evolution of perturbation $(a)$ density, $(b)$ radial velocity, $(c)$ temperature, $(d)$ entropy : ,$- \tau=\tau_{1} ;----, \tau=0.217 ;-\cdots, \tau=0.277 ; \cdots \cdots, \tau=0.517 ;---, \tau=0.837 ;-, \tau=$ $1.477 ;----, \tau=5.317 ;-.-, \tau=10.437$.

section, the $1 / \tilde{r}$ decay in velocity is not necessary to generate radial inflow - any swirling axisymmetric compressible flow generates a radial inflow due to viscous effects.

For both initial conditions studied in the previous sections, the Oseen vortex produced entropy far from the core, and gained mass due to a negative radial velocity far from the core. For an arbitrary swirling flow, to $O\left(M^{2}\right)$, the total change in entropy of the vortex is given by integrating (4.39) over the entire area of the vortex :

$$
\frac{\partial}{\partial \tau} \int_{0}^{\infty} \tilde{s}_{1} \tilde{r} \mathrm{~d} \tilde{r}=\int_{0}^{\infty}(\gamma-1)\left(\frac{\partial v_{0}}{\partial \tilde{r}}-\frac{\tilde{v}_{0}}{\tilde{r}}\right)^{2} \tilde{r} \mathrm{~d} \tilde{r}
$$

(Owing to the multiple lengthscales (acoustic and viscous) in the problem, the upper limits in these integrals must be interpreted as the outer limit of the inner variable, $\left(a_{\infty} t\right) / L$, where $L$ is the (time-dependent) location of the maximum tangential velocity.) Equation (4.15) shows that the total entropy of the vortex increases in time, regardless of the initial distribution of vorticity, density and entropy. For vortices with finite circulation, the total change in entropy increases as $\ln \tau$, while for higher vorticity modes $((m>0)$ in $(4.12))$ the total entropy increases at a rate proportional to $1 / \tau^{2 m}$.

The thermal energy equation, to $O\left(M^{2}\right)$, is

$$
\frac{\partial}{\partial \tau} \int_{0}^{\infty} \tilde{T}_{1} \tilde{r} \mathrm{~d} \tilde{r}=\int_{0}^{\infty}(\gamma-1) \frac{\partial \tilde{p}_{0}}{\partial \tau} \tilde{r} \mathrm{~d} \tilde{r}+\int_{0}^{\infty}(\gamma-1)\left(\frac{\partial \tilde{v}_{0}}{\partial \tilde{r}}-\frac{\tilde{v}_{0}}{\tilde{r}}\right)^{2} \tilde{r} \mathrm{~d} \tilde{r} .
$$

Physically, equation (4.42) gives the increase in total enthalpy of the fluid due to pressure work and viscous dissipation. But since the radial pressure gradient of the vortex must be provided by the centripetal acceleration, the pressure work term in $(4.42)$ is given, by integration by parts of $(4.9 d)$, by

$$
\int_{0}^{\infty} \frac{\partial \tilde{p}_{0}}{\partial \tau} \tilde{r} \mathrm{~d} \bar{r}=-\frac{\partial}{\partial \tau} \int_{0}^{\infty} \frac{\tilde{v}_{0}^{2}}{2} \tilde{r} \mathrm{~d} \tilde{r}
$$


Therefore the pressure work term in the thermal energy equation is opposite in sign but equal in magnitude to the change in kinetic energy of the vortex. Since the kinetic energy of the vortex decreases as the vortex decays, the pressure work term is positive, and hence the vortex is compressed. Substituting (4.43) and using (4.14a), $(4.14 e)$ in (4.42) gives the necessary radial velocity at infinity for such a compression :

$$
\lim _{\tilde{r} \rightarrow \infty} \tilde{u}_{1} r=-(2-\gamma) \int_{0}^{\infty}\left(\frac{\partial \tilde{v}_{0}}{\partial \tilde{r}}-\frac{\tilde{v}_{0}}{\tilde{r}}\right)^{2} \tilde{r} \mathrm{~d} \tilde{r} .
$$

Hence the compressible axisymmetric vortex has a negative radial velocity far from the core, regardless of the initial distributions of vorticity, density and entropy.

The specific form of the radial velocity can be found by substituting (4.12) into (4.44). For the Oseen vortex $\left(C_{0} \neq 0, C_{n}=0, n=1,2, \ldots\right)$, far from the core

$$
\tilde{u}_{1} \sim-\frac{C_{0}^{2}(2-\gamma)}{\overline{\operatorname{Re} \tau \tilde{r}}}
$$

If $v_{\mathrm{m}}=v_{\mathrm{m}}(t)$ is chosen to be the maximum velocity of the vortex at time $t$, and $L(t)$ the radius where the maximum occurs then (4.45) can be written

$$
\frac{u}{v_{\mathrm{m}}(t)} \sim-\frac{\alpha M^{* 2}(2-\gamma)}{R e^{*} r^{*}}
$$

where $M^{*}=v_{\mathrm{m}}(t) / a_{\infty}, R e^{*}=\Gamma / \nu, r^{*}=r / L(t)$, and $\alpha \approx 3.433$ is a constant. Thus the radial velocity only depends on the local (in time) characteristics of the vortex. Far from the core $v \sim \Gamma / 2 \pi r$ and the ratio of the radial velocity to tangential velocity is

$$
\frac{u}{v} \sim-\frac{2 \pi \beta M^{* 2}(2-\gamma)}{R e^{*}}
$$

where $\beta \approx 2.455$ is a constant. Equation (4.47) shows that $u / v \ll 1$ when $M^{* 2} / R e^{*} \ll$ 1 , which is consistent with the multiple-timescale and low-Mach-number expansions. For the Taylor swirl $\left(C_{0}=0, C_{1} \neq 0, C_{n}=0, n=2,3, \ldots\right)$ the radial velocity far from the core is

$$
\tilde{u}_{1} \sim-\frac{C_{1}^{2}(2-\gamma)}{2 \tau^{3} \tilde{r}}
$$

When scaled with the (time-dependent) Mach and Reynolds numbers, the radial velocity is

$$
\frac{u}{v_{\mathrm{m}}(t)} \sim-\frac{\kappa M^{* 2}(2-\gamma)}{R e^{*} r^{*}},
$$

where $M^{*}=v_{\mathrm{m}}(t) / a_{\infty}, R e^{*}=\mathscr{M} /\left(L(t)^{2} \nu\right), r^{*}=r / L(t)$, and $\kappa \approx 4.482$ is a constant. $\mathscr{M}$ is the (constant) total angular momentum as defined by (1.7). The radial velocity is again dependent on only the local characteristics of the vortex.

\section{Conclusions}

Analytical solutions for the evolution of a free, compressible, viscous, heatconducting vortex are found in the limit of large, but finite, Reynolds number, and small, but finite Mach-number. The full equations of motion for two-dimensional axisymmetric flow are expanded on an acoustic and a viscous timescale. An ordinary differential equation giving the dependence of the radial velocity on the tangential 
velocity, density, and temperature of the compressible vortex is derived. Estimates of the radial velocity, found by integrating this equation, are in good agreement with numerical solutions of the full equations. Agreement with the experiments of M \& B is poor, and the discrepancy is attributed to three-dimensionality in the experiments. It is shown that the profiles reported by $M \& B$ violate the Second Law of Thermodynamics for two-dimensional axisymmetric flow. The radial velocity in the two-dimensional compressible vortex is generated by viscous effects. It is small $\left(\sim M^{2} / R e\right)$ and hence does not play an important role in the decay of the vorticity over long times.

The equations for the evolution of the vortex on the viscous timescale are expanded in powers of $M^{2}$. The base flow $(O(1))$ is chosen to be an Oseen vortex. $O\left(M^{2}\right)$ corrections for the density, temperature, entropy and radial velocities are found for a vortex which is initially homentropic, and for a vortex which initially has a uniform density.

In the case of an initially homentropic vortex, the density decays like $1 / r^{2}$ far from the vortex core. The entropy far from the core is proportional to $1 / r^{4}$ and increases with time. This entropy production is the result of viscous dissipation and heat conduction produced by the $1 / r$ decay of the Oseen tangential velocity profile. In the vortex core, the entropy is found to first increase with time, and eventually decay back to zero as the core of the vortex spreads. In the case of a vortex which initially has uniform density, the entropy decays like $1 / r^{2}$ far from the vortex core. The entropy changes in time to $O\left(1 / r^{4}\right)$, again due to viscous effects. The density at first decreases in the core, but later builds up there due to the negative radial velocity induced by viscous effects outside the core. Eventually the spreading of the core changes the sign of the radial velocity and an outflow of mass allows the density in the core to decay to ambient conditions.

The analysis shows that, to $O\left(M^{2}\right)$, compressible axisymmetric swirling flows are compressed, regardless of the initial distributions of vorticity, density, and entropy. The compression is the result of pressure work which is required to balance the viscously decaying kinetic energy of the vortex. Mass is convected into the compressible vortex by a small negative radial velocity far from the vortex core. The radial velocity is described in terms of an arbitrary distribution of tangential velocity. When scaled with the (time-dependent) maximum tangential velocity of the vortex, the radial velocity is proportional to $M^{* 2} /\left(r^{*} R e^{*}\right)$, where $M^{*}$ and $R e^{*}$ are the relevant (time-dependent) Mach and Reynolds numbers of the swirling flow, and $r^{*}$ is the distance from the core relative to the location where the maximum tangential velocity occurs.

This work was supported by the Office of Naval Research under contract number ONR-N00014-88-K-0592. The computer time was provided by NASA Ames Research Center.

\section{REFERENCES}

Abramowitz, M. A. \& Stegun, I. A. 1972 Handbook of Mathematical Functions. Dover.

BeLlamy-Knights, P. G. 1980 Viscous compressible heat conducting spiraling flow. Q. J. Mech. Appl. Maths 33, 321-336.

Bershader, D. 1988 Shock tube studies of vortex structure and behavior. Shock Tubes and Waves: Proc. Sixteenth Intl Symp. on Shock Tubes and Waves, pp. 5-18. VCH Verlagsgesellschaft.

Colonius, T., Lele, S. K. \& Motn, P. 1991 Scattering of sound waves by a compressible vortex. AIAA Paper 91-0494.

Ingropera, F. P. \& DeWitT, D. P. 1985 Introduction to Heat Transfer. John Wiley \& Sons. 
LELE, S. K. 1990 Compact finite difference schemes with spectral-like resolution. CTR Manuscript 107. Stanford University; J. Comput. Phys. (submitted).

Long, R. R. 1961 A vortex in an infinite viscous fluid. J. Fluid Mech. 11, 611-624.

MaCK, L. M. 1960 The compressible viscous heat-conducting vortex. J. Fluid Mech. 8, 284-292.

MANDella, M. J. 1987 Experimental and analytical studies of compressible vortices. Ph.D. thesis, Stanford University.

Mandella, M., Moon, Y.J. \& Bershader, D. 1986 Quantitative study of shock generated compressible vortex flows. In Shock Waves and Shock Tubes (ed. D. Bershader \& R. Hanson), pp. 471-477. Stanford University Press.

Merzkirch, W. 1964 Theoretische und experimentelle Untersuchungen an einer instationären Wirbelströmung. Z. Flugwiss. 12, 395-401.

Morton, B. R. 1969 The strength of vortex and swirling core flows. J. Fluid Mech. 38, 315-333.

Neurville, A. De 1957 The dying vortex. In Proc. Fifth Midwestern Conf, on Fluid Mechanics, p. 365. University of Michigan.

Oseen, C. W. 1912 Über Wirbelbewegung in einer reibenden Flüssigkeit. Ark.f. Mat. Astron. Fys. 7,14

Rotr, N. 1959 On the viscous core of a line vortex II. Z. Angew. Math. Phys. 10, 73-81.

RotT, N. \& Lewellen, W. S. 1966 Boundary layers and their interactions in rotating flows. Prog. Aeronaut. Sci. 7, 111-144.

Sibulkis, M. 1961 Unsteady, viscous, circular flow. Part 1. The line impulse of angular momentum. J. Fluid Mech. 11, 291-308.

Taylor, G. I. 1918 On the dissipation of eddies. Aero. Res. Comm., $R$ and $M 598$.

TAYLOR, G. I. 1930 Recent work on the flow of compressible fluids. J. Lond. Math. Soc. 5, 224-240.

'Tномpson, K. W. 1987 Time dependent boundary conditions for hyperbolic systems. J. Comput. Phys. 68, 1-24.

Uberor, M. S. 1979 Mechanisms of decay of laminar and turbulent vortices. J. Fluid Mech. 90 , 241-255. 